\title{
ON THE DYNAMICAL BEHAVIOR OF THE ABC MODEL
}

\author{
LORENZO BERTINI, NICOLETTA CANCRINI, AND GUSTAVO POSTA
}

\begin{abstract}
We consider the ABC dynamics, with equal density of the three species, on the discrete ring with $N$ sites. In this case, the process is reversible with respect to a Gibbs measure with a mean field interaction that undergoes a second order phase transition. We analyze the relaxation time of the dynamics and show that at high temperature it grows at most as $N^{2}$ while it grows at least as $N^{3}$ at low temperature.
\end{abstract}

\section{INTRODUCTION}

The $A B C$ model, introduced by Evans et al. 14, 15, is a one-dimensional stochastic conservative dynamics with local jump rates whose invariant measure undergoes a phase transition. It is a system consisting of three species of particles, traditionally labeled $A, B, C$, on a discrete ring with $N$ sites. The system evolves by nearest neighbor particles exchanges with the following (asymmetric) rates: $A B \rightarrow B A$, $B C \rightarrow C B, C A \rightarrow A C$ with rate $q \in(0,1]$ and $B A \rightarrow A B, C B \rightarrow B C, A C \rightarrow C A$ with rate $1 / q$. In particular, the total numbers of particles $N_{\alpha}, \alpha \in\{A, B, C\}$, of each species are conserved and satisfy $N_{A}+N_{B}+N_{C}=N$. Observe that the case $q=1$ corresponds to a three state version of the symmetric simple exclusion process. When $q \in(0,1)$, Evans et al. 14, 15] argued that in the thermodynamic limit $N \rightarrow \infty$ with $N_{\alpha} / N \rightarrow r_{\alpha}$ the system segregates into pure $A, B$, and $C$ regions, with translationally invariant distribution of the phase boundaries. In the equal densities case $N_{A}=N_{B}=N_{C}=N / 3$ the dynamics is reversible and its invariant measure can be explicitly computed. As shown in [16, 17, the ABC model can be reformulated terms of a dynamic of random walks on the triangular lattice.

As discussed by Clincy et al. 9, the natural scaling to investigate the asymptotic behavior of the ABC model is the weakly asymmetric regime $q=\exp \left\{-\frac{\beta}{2 N}\right\}$, where the parameter $\beta \in[0,+\infty)$ plays the role of an inverse temperature. With this choice the reversible measure of the equal densities case $r_{A}=r_{B}=r_{C}=1 / 3$ becomes a canonical Gibbs measure, that we denote by $\nu_{N}^{\beta}$, with a mean field Hamiltonian. The measure $\nu_{N}^{\beta}$ undergoes a second order phase transition at $\beta_{\mathrm{c}}=2 \pi \sqrt{3} \approx$ 10.88. This phase transition has been further analyzed in [1] and it is described in terms of the free energy functional $\mathcal{F}_{\beta}$ associated to $\nu_{N}^{\beta}$. The functional $\mathcal{F}_{\beta}$ is (apart an additive constant) the large deviations rate function for $\nu_{N}^{\beta}$ in the scaling limit in which the discrete ring with $N$ sites is embedded in the one-dimensional torus and the particles configuration is described in terms of the corresponding densities profiles $\left(\rho_{A}, \rho_{B}, \rho_{C}\right)$. In this limit, $\mathcal{F}_{\beta}\left(\rho_{A}, \rho_{B}, \rho_{C}\right)$ thus gives the asymptotic probability of observing the density profile $\left(\rho_{A}, \rho_{B}, \rho_{C}\right)$. In particular, the minimizer of $\mathcal{F}_{\beta}$ describes the typical behavior of the system as $N \rightarrow \infty$. The phase transition

2000 Mathematics Subject Classification. Primary 60K35, 82C20; Secondary 82C22, 60B15.

Key words and phrases. ABC model, Spectral gap, Mean field Gibbs measures, Interchange process. 
of $\nu_{N}^{\beta}$ corresponds to the following behavior of the free energy functional [1, 9, 16]. For $\beta \in\left[0, \beta_{\mathrm{c}}\right]$ the minimum of $\mathcal{F}_{\beta}$ is uniquely achieved at the homogeneous profile $(1 / 3,1 / 3,1 / 3)$. For $\beta>\beta_{\mathrm{c}}$ the functional $\mathcal{F}_{\beta}$ has a continuum of minimizers, parameterized by the translations, which describes the phase segregation. As shown in 4, this phase transition can also be detected via the two-point correlation functions of $\nu_{N}^{\beta}$ which become singular when the system approaches the transition. As shown in [1, 16], for $\beta>2 \beta_{\mathrm{c}}$ the functional $\mathcal{F}_{\beta}$ has other critical points besides the homogeneous profile and the one-parameter family of minimizers.

For unequal densities the invariant measure of the $\mathrm{ABC}$ dynamics on a ring is not reversible, that is the stationary state is no longer an equilibrium state, and cannot be computed explicitly. As discussed in [4, 9], a stability analysis of the homogeneous density profile shows that for $\beta>2 \pi\left[1-2\left(r_{A}^{2}+r_{B}^{2}+r_{C}^{2}\right)\right]^{-1 / 2}$ it becomes unstable. As stated there, one however expects that the phase transition, at least for particular values of the parameters $r_{A}, r_{B}, r_{C}$, becomes of the first order. Again in 4, 9, the asymptotic of the two-point correlation functions is computed in the homogeneous phase and the large deviation rate function $\mathcal{F}_{\beta}$ has been calculated up to order $\beta^{2}$. When the ABC dynamics is considered on an open interval with reflecting endpoints, the corresponding invariant measure is reversible for all values of the densities [1. In particular, it has the same Gibbs form as the one in the ring for the equal density case.

The main purpose of the present paper is the discussion of the phase transition of the ABC model on a ring with $N$ sites from a dynamical viewpoint. More precisely, we focus on the asymptotic behavior, as $N$ diverges, of the relaxation time $\tau_{N}^{\beta}$ which measures the time the dynamics needs to reach the stationary probability. Our analysis is restricted to the equal density case $r_{A}=r_{B}=r_{C}=1 / 3$ in which the invariant measure $\nu_{N}^{\beta}$ is explicitly known and reversible. As usual, the relaxation time $\tau_{N}^{\beta}$ is defined as the inverse of the spectral gap of the generator $L_{N}^{\beta}$ of the underlying Markov process. Observe that, in view of the reversibility, $L_{N}^{\beta}$ is a selfadjoint operator on $L^{2}\left(d \nu_{N}^{\beta}\right)$. Our main result implies that the asymptotic behavior of the relaxation time $\tau_{N}^{\beta}$ reflects the phase transition of the corresponding stationary measure $\nu_{N}^{\beta}$. We indeed show that for $\beta$ small enough $\tau_{N}^{\beta}$ is at most of order $N^{2}$ while for $\beta>\beta_{\mathrm{c}}$ it is at least of order $N^{3}$.

The diffusive behavior $\tau_{N}^{\beta} \sim N^{2}$ is characteristic of conservative dynamics in the high temperature regime, the typical example being the Kawasaki dynamics for the Ising model. Indeed, this has been proven by different techniques in several contexts, see e.g. [5, 7, 22]. We here follow the approach introduced in [5] which is based upon a perturbative argument in $\beta$ and can be directly applied to the case of mean field interactions. On the other hand, the behavior $\tau_{N}^{\beta} \sim N^{3}$ in the supercritical regime is characteristic of the system under consideration; we briefly discuss the heuristic picture. As discussed in 9] and then also in [4, at time $O\left(N^{2}\right)$ the densities profiles of the three species $\left(\rho_{A}, \rho_{B}, \rho_{C}\right)$ evolve according to the hydrodynamic equations

$$
\begin{aligned}
& \partial_{t} \rho_{A}+\beta \nabla\left[\rho_{A}\left(\rho_{C}-\rho_{B}\right)\right]=\Delta \rho_{A} \\
& \partial_{t} \rho_{B}+\beta \nabla\left[\rho_{B}\left(\rho_{A}-\rho_{C}\right)\right]=\Delta \rho_{B} \\
& \partial_{t} \rho_{C}+\beta \nabla\left[\rho_{C}\left(\rho_{B}-\rho_{A}\right)\right]=\Delta \rho_{C}
\end{aligned}
$$


where $\nabla$ and $\Delta$ denote the gradient and Laplacian on the continuous torus, respectively. As follows from microscopic reversibility, the evolution (1.1) can be obtained as a suitable gradient flow of the free energy $\mathcal{F}_{\beta}$. In particular, while the homogeneous profile $(1 / 3,1 / 3,1 / 3)$ is the unique, globally attractive, stationary solution to (1.1) for $\beta<\beta_{\mathrm{c}}$, the (one parameter family of) minimizers of $\mathcal{F}_{\beta}$ are stationary solutions to (1.1) when $\beta>\beta_{\mathrm{c}}$. According to the fluctuating hydrodynamic theory, we argue that, for large but finite $N$, the hydrodynamic equation (1.1) gives an accurate description of the system provided one adds in (1.1) a suitable noise term $O(1 / \sqrt{N})$. At time $O\left(N^{2}\right)$ the $\mathrm{ABC}$ dynamics then behaves as a Brownian motion on the set of minimizers of $\mathcal{F}_{\beta}$ with diffusion coefficient proportional to $1 / N$, see [3. The time to thermalize is thus $O\left(N^{3}\right)$.

The above scenario accounts for the correct asymptotics of the relaxation time as long as there are no other local minima of $\mathcal{F}_{\beta}$. As proven in [1, this is certainly the case for $\beta \in\left(\beta_{\mathrm{c}}, 2 \beta_{\mathrm{c}}\right]$. On the other hand, for $\beta>2 \beta_{\mathrm{c}}$ other critical points of $\mathcal{F}_{\beta}$ do appear but it is not known if they correspond to local minima (this is indeed an open question in [1]). Were they local minima the ABC model would exhibit a metastable behavior, i.e. starting in a neighborhood of such local minima the process would spend a time exponential in $N$ in that neighborhood before reaching the global minimizer. Numerical evidences [18] suggest such metastable behavior which would imply $\tau_{N}^{\beta} \sim \exp \{c N\}$ for $\beta>2 \beta_{\text {c }}$.

\section{Notation AND RESUlts}

The ABC process. Given a positive integer $N$, we let $\mathbf{Z}_{N}=\{0, \cdots, N-1\}$ be the ring of the integers modulo $N$. The configuration space with $N$ sites is $\widetilde{\Omega}_{N}:=\{A, B, C\}^{\mathbf{Z}_{N}}$, elements of $\widetilde{\Omega}_{N}$ are denoted by $\zeta$, for $x \in \mathbf{Z}_{N}$ the species of the particle at the site $x$ is thus $\zeta(x) \in\{A, B, C\}$. We also let $\eta_{\alpha}: \widetilde{\Omega}_{N} \rightarrow\{0,1\} \mathbf{Z}_{N}$, $\alpha \in\{A, B, C\}$, be the $\alpha$ occupation numbers namely, $\left[\eta_{\alpha}(\zeta)\right](x):=\mathbf{1}_{\{\alpha\}}(\zeta(x))$ in which $\mathbf{1}_{E}$ stands for the indicator function of the set $E$. Note that for each $x \in \mathbf{Z}_{N}$ we have $\eta_{A}(x)+\eta_{B}(x)+\eta_{C}(x)=1$. Whereas $\eta=\left(\eta_{A}, \eta_{B}, \eta_{C}\right)$ is a function of the configuration $\zeta$ we shall omit to write explicitly the dependence on $\zeta$.

Given $x, y \in \mathbf{Z}_{N}$ and $\zeta \in \widetilde{\Omega}_{N}$ we denote by $\zeta^{x, y}$ the configuration obtained from $\zeta$ by exchanging the particles at the sites $x$ and $y$, i.e.

$$
\left(\zeta^{x, y}\right)(z):= \begin{cases}\zeta(y) & \text { if } z=x \\ \zeta(x) & \text { if } z=y \\ \zeta(z) & \text { otherwise }\end{cases}
$$

The ABC process is the continuous time Markov chain on the state space $\widetilde{\Omega}_{N}$ whose generator $L_{N}=L_{N}^{\beta}$ acts on functions $f: \widetilde{\Omega}_{N} \rightarrow \mathbf{R}$ as

$$
L_{N}^{\beta} f(\zeta)=\sum_{x \in \mathbf{Z}_{N}} c_{x}^{\beta}(\zeta)\left[f\left(\zeta^{x, x+1}\right)-f(\zeta)\right]
$$

where, for $\beta \geq 0$ the jump rates $c_{x}^{\beta}=c_{x}^{\beta, N}$ are given by

$$
c_{x}^{\beta, N}(\zeta):= \begin{cases}\exp \left\{-\frac{\beta}{2 N}\right\} & \text { if }(\zeta(x), \zeta(x+1)) \in\{(A, C),(C, B),(B, A)\} \\ \exp \left\{\frac{\beta}{2 N}\right\} & \text { otherwise. }\end{cases}
$$

As follows from (2.2), the total number of particles of each species is conserved. Therefore, given three positive integers $N_{\alpha}, \alpha \in\{A, B, C\}$ such that $N_{A}+N_{B}+$ 
$N_{C}=N$, we have a well defined process on the linear manifold $\sum_{x \in \mathbf{Z}_{N}} \eta_{\alpha}(x)=N_{\alpha}$, $\alpha \in\{A, B, C\}$. The ABC dynamics is irreducible when restricted to such manifold; hence the process is ergodic and admits a unique invariant measure. In the case $\beta=0$ this measure is the uniform probability. On the other hand, when $\beta>0$ the explicit expression of the invariant measure is in general not known. However, as we next discuss, in the case $N_{A}=N_{B}=N_{C}$ the ABC process satisfies the detailed balance condition with respect to a mean field Gibbs measure [14, 15.

Invariant measure in the equal densities case. We assume that $N$ is a multiple of 3 and we restrict to the case in which $N_{A}=N_{B}=N_{C}$. We shall then consider the $\mathrm{ABC}$ process on

$$
\Omega_{N}:=\left\{\zeta \in \widetilde{\Omega}_{N}: \sum_{x \in \mathbf{Z}_{N}} \eta_{A}(x)=\sum_{x \in \mathbf{Z}_{N}} \eta_{B}(x)=\sum_{x \in \mathbf{Z}_{N}} \eta_{C}(x)=\frac{N}{3}\right\} .
$$

The Hamiltonian $H_{N}: \Omega_{N} \rightarrow \mathbf{R}$ is defined by

$$
H_{N}(\zeta):=\frac{1}{N^{2}} \sum_{0 \leq x<y \leq N-1}\left[\eta_{A}(x) \eta_{C}(y)+\eta_{B}(x) \eta_{A}(y)+\eta_{C}(x) \eta_{B}(y)\right] .
$$

In view of the equal densities constraint, an elementary computation shows that the right hand side above does not depend on the choice of the origin. Equivalently, $H_{N}$ is a translation invariant function on $\Omega_{N}$. Given $\beta \geq 0$, we denote by $\nu_{N}^{\beta}$ the probability measure on $\Omega_{N}$ defined by

$$
\nu_{N}^{\beta}(\zeta):=\frac{1}{Z_{N}^{\beta}} \exp \left\{-\beta N H_{N}(\zeta)\right\}
$$

where $Z_{N}^{\beta}$, the partition function, is the proper normalization constant. In the sequel, given a function $f$ on $\Omega_{N}$ we denote respectively by $\nu_{N}^{\beta}(f)$ and $\nu_{N}^{\beta}(f, f)$ the expectation and variance of $f$ with respect to $\nu_{N}^{\beta}$.

As observed in [14, 15, the ABC process is reversible with respect to $\nu_{N}^{\beta}$. In other worlds, the generator $L_{N}^{\beta}$ in (2.2) is a selfadjoint operator on $L^{2}\left(\Omega_{N}, d \nu_{N}^{\beta}\right)$ and in particular $\nu_{N}^{\beta}$ is the invariant measure.

Asymptotics of the spectral gap. The spectrum of $L_{N}^{\beta}$ in (2.2), considered as a selfadjoint operator on $L^{2}\left(\Omega_{N}, \nu_{N}^{\beta}\right)$, is a finite subset of the negative real axes and, in view of the ergodicity of the associated process, zero is a simple eigenvalue of $L_{N}^{\beta}$. The spectral gap of $L_{N}^{\beta}$, denoted by $\operatorname{gap}\left(L_{N}^{\beta}\right)$, is the absolute value of the second largest eigenvalue. The spectral gap can be characterized in variational terms as follows: $\operatorname{gap}\left(L_{N}^{\beta}\right)$ is largest constant $\lambda \geq 0$ such that the Poincaré inequality

$$
\lambda \nu_{N}^{\beta}(f, f) \leq \nu_{N}^{\beta}\left(f\left(-L_{N}^{\beta}\right) f\right)
$$

holds for any $f \in L^{2}\left(\Omega_{N}, d \nu_{N}^{\beta}\right)$. The spectral gap controls the speed of convergence to equilibrium of the associated process in the following sense. For each $f \in L^{2}\left(\Omega_{N}, d \nu_{N}^{\beta}\right)$

$$
\nu_{N}^{\beta}\left(e^{t L_{N}^{\beta}} f, e^{t L_{N}^{\beta}} f\right) \leq e^{-2 \operatorname{gap}\left(L_{N}^{\beta}\right) t} \nu_{N}^{\beta}(f, f) .
$$

Our main result concerns the asymptotic behavior of gap $\left(L_{N}^{\beta}\right)$ as $N$ diverges. In particular we show this behavior differs in the subcritical and supercritical regimes. 


\section{Theorem 2.1.}

(i) There exist constants $\beta_{0}, C_{0}>0$ such that for any $\beta \in\left[0, \beta_{0}\right]$ and any $N$

$$
\operatorname{gap}\left(L_{N}^{\beta}\right) \geq C_{0} \frac{1}{N^{2}}
$$

(ii) Let $\beta_{\mathrm{c}}:=2 \pi \sqrt{3}$. For each $\beta>\beta_{\mathrm{c}}$ there exists a constant $C(\beta)>0$ such that for any $N$

$$
\operatorname{gap}\left(L_{N}^{\beta}\right) \leq C(\beta) \frac{1}{N^{3}} .
$$

The above statement raises few natural issues. As discussed in the Introduction, the $1 / N^{2}$ asymptotic of the spectral gap is a common feature of conservative stochastic dynamics in the high temperature regime. Indeed, as proven in 24] for the simple exclusion process and in [7, 22] for high temperature Kawasaki dynamics, the spectral gap admits an upper bound that matches (2.8). We expect that the diffusive behavior $\operatorname{gap}\left(L_{N}^{\beta}\right)=O\left(1 / N^{2}\right)$ holds for any $\beta \in\left[0, \beta_{\mathrm{c}}\right)$. The methods used in the present paper are based on a perturbation argument around $\beta=0$ and their extension to the whole subcritical regime does not appear feasible. In principle, the techniques developed in [7, 22, which require as an input a strong spatial mixing of the stationary probability, can be applied up to the critical temperature. Those techniques have been however developed for short range interactions and they do not seem, at least directly, applicable to mean field Hamiltonians.

Another, somehow more fundamental, issue is whether $1 / N^{3}$ is the right scaling of the spectral gap in the supercritical regime. We expect that this is the correct scaling for $\beta$ between $\beta_{\mathrm{c}}$ and $2 \beta_{\mathrm{c}}$. We mention that this behavior is also the one expected for the Kawasaki dynamics for the low temperature two dimensional Ising model with plus boundary condition (pure state) [6. Indeed, in this case the heuristic picture presented in the Introduction corresponds to the diffusion of the Wulff bubble. While the statement (ii) in Theorem 2.1 is proven by exhibiting a suitable slowly varying test function, a proof of a matching lower bound appears considerably harder. The ABC model is however much simpler than short range models and it therefore might be a useful starting point toward the understanding of conservative dynamics in the phase transition region.

For $\beta$ larger than $2 \beta_{\mathrm{c}}$, a preliminary question is whether the other critical points of $\mathcal{F}_{\beta}$ correspond to local minima. In such a case it is possible to construct a slowly varying test function which yields the upper bound $\operatorname{gap}\left(L_{N}^{\beta}\right) \leq \exp \{-c N\}$ for some constant $c=c(\beta)>0$. Observe that the general argument in [6] gives for free the lower bound $\operatorname{gap}\left(L_{N}^{\beta}\right) \geq \exp \{-C N\}$ for some $C=C(\beta)<+\infty$.

We finally discuss the behavior of the spectral gap of the ABC process on an interval with zero flux condition at the endpoints. As shown in 1, in such a case the process is reversible with respect to a mean field Gibbs probability for all values of the densities. In the high temperature regime $\beta \ll 1$, the methods here developed can be directly applied to get the diffusive behavior $1 / N^{2}$. As far as the low temperature regime is concerned, the case of equal densities is the same as the one on the ring and we can therefore conclude that the upper bound $1 / N^{3}$ holds also in this setting. 


\section{Asymptotics of the GibBs measure}

The upper bound on the spectral gap in the supercritical regime requires the law of large numbers for the empirical density with respect to the Gibbs measure $\nu_{N}^{\beta}$. This result is proven by combining the large deviations principle for $\nu_{N}^{\beta}$ with the analysis of the minimizers of the free energy in 1]. As $\nu_{N}^{\beta}$ is a Gibbs measure with a mean field interaction, the associated large deviations principle can be proven by standard tools. The specific application to the ABC model has not however been detailed in the literature, we thus present here the whole argument.

Empirical density. We let $\mathbf{T}:=\mathbf{R} / \mathbf{Z}$ be the one-dimensional torus of side length one; the coordinate on $\mathbf{T}$ is denoted by $r \in[0,1)$. The inner product in $L^{2}\left(\mathbf{T}, d r ; \mathbf{R}^{3}\right)$ is denoted by $\langle\cdot, \cdot\rangle$. We set $\widetilde{\mathcal{M}}:=L^{\infty}\left(\mathbf{T}, d r ;[0,1]^{3}\right)$ and denote by $\rho=\left(\rho_{A}, \rho_{B}, \rho_{C}\right)$ its elements. We consider $\widetilde{\mathcal{M}}$ endowed with the weak* topology. Namely, a sequence $\left\{\rho^{n}\right\}$ converges to $\rho$ in $\widetilde{\mathcal{M}}$ iff $\left\langle\rho^{n}, \phi\right\rangle \rightarrow\langle\rho, \phi\rangle$ for any function $\phi \in L^{1}\left(\mathbf{T}, d r ; \mathbf{R}^{3}\right)$, equivalently for any smooth function $\phi \in C^{\infty}\left(\mathbf{T} ; \mathbf{R}^{3}\right)$. Note that $\widetilde{\mathcal{M}}$ is a compact Polish space, i.e. separable, metrizable, and complete.

We introduce

$$
\mathcal{M}:=\left\{\rho \in \widetilde{\mathcal{M}}: \rho_{A}+\rho_{B}+\rho_{C}=1, \int_{0}^{1} d r \rho_{\alpha}(r)=\frac{1}{3}, \alpha \in\{A, B, C\}\right\}
$$

noticing it is a closed subset of $\widetilde{\mathcal{M}}$ that we consider equipped with the relative topology and the associated Borel $\sigma$-algebra. The set of Borel probability measures on $\mathcal{M}$, denoted by $\mathcal{P}(\mathcal{M})$, is endowed with the topology induced by the weak convergence of probability measures; namely, $\mathcal{P}_{n} \rightarrow \mathcal{P}$ iff for each continuous $F: \mathcal{M} \rightarrow \mathbf{R}$ we have $\int d \mathcal{P}_{n} F \rightarrow \int d \mathcal{P} F$. Note that also $\mathcal{P}(\mathcal{M})$ is a compact Polish space.

We define the empirical density as the map $\pi_{N}: \Omega_{N} \rightarrow \mathcal{M}$ given by

$$
\pi_{N}(\zeta)(r):=\sum_{x \in \mathbf{Z}_{N}} \eta(x) \mathbf{1}_{[x / N,(x+1) / N)}(r), \quad r \in \mathbf{T},
$$

recall $\eta$ is the map defined at the beginning of Section 2, We set $\mathcal{P}_{N}^{\beta}:=\nu_{N}^{\beta} \circ \pi_{N}^{-1}$ namely, $\mathcal{P}_{N}^{\beta}$ is the law of $\pi_{N}$ when $\zeta$ is distributed according to $\nu_{N}^{\beta}$. Note that $\left\{\mathcal{P}_{N}^{\beta}\right\}$ is a sequence in $\mathcal{P}(\mathcal{M})$.

Large deviations principle. The entropy (with a sign convention opposite to the standard one in physical literature) is the convex lower semicontinuous functional $\mathcal{S}: \mathcal{M} \rightarrow[0,+\infty)$ defined by

$$
\mathcal{S}(\rho):=\int_{0}^{1} d r\left[\rho_{A}(r) \log \frac{\rho_{A}(r)}{1 / 3}+\rho_{B}(r) \log \frac{\rho_{B}(r)}{1 / 3}+\rho_{C}(r) \log \frac{\rho_{C}(r)}{1 / 3}\right]
$$

and the energy is the continuous functional $\mathcal{H}: \mathcal{M} \rightarrow \mathbf{R}$ defined by

$$
\mathcal{H}(\rho):=\int_{0}^{1} d r \int_{r}^{1} d r^{\prime}\left[\rho_{A}(r) \rho_{C}\left(r^{\prime}\right)+\rho_{B}(r) \rho_{A}\left(r^{\prime}\right)+\rho_{C}(r) \rho_{B}\left(r^{\prime}\right)\right] .
$$

For $\beta \geq 0$ the free energy (in which we omit the prefactor $1 / \beta$ ) is finally the functional $\mathcal{F}_{\beta}: \mathcal{M} \rightarrow \mathbf{R}$ defined by

$$
\mathcal{F}_{\beta}:=\mathcal{S}+\beta \mathcal{H}
$$


Theorem 3.1. The sequence $\left\{\mathcal{P}_{N}^{\beta}\right\}$ satisfies a large deviation principle with rate function $\mathcal{I}_{\beta}=\mathcal{F}_{\beta}-\inf \mathcal{F}_{\beta}$. Namely, for each closed set $\mathcal{C} \subset \mathcal{M}$ and each open set $\mathcal{O} \subset \mathcal{M}$

$$
\begin{aligned}
& \varlimsup_{N \rightarrow \infty} \frac{1}{N} \log \mathcal{P}_{N}^{\beta}(\mathcal{C}) \leq-\inf _{\rho \in \mathcal{C}} \mathcal{I}_{\beta}(\rho) \\
& \varliminf_{N \rightarrow \infty} \frac{1}{N} \log \mathcal{P}_{N}^{\beta}(\mathcal{O}) \geq-\inf _{\rho \in \mathcal{O}} \mathcal{I}_{\beta}(\rho) .
\end{aligned}
$$

Since the beautiful Lanford's lectures [21, large deviations principles for Gibbs measures has become a basic topic in equilibrium statistical mechanics, see in particular [13] for the case of mean field interactions. On the other hand, the current setting is not completely standard as we are looking to large deviations of the empirical density for canonical Gibbs measures. We therefore give a detailed proof of the above result. The first step is the large deviations principle when $\beta=0$; recall that $\mathcal{P}_{N}^{0}=\nu_{N}^{0} \circ \pi_{N}^{-1}$ is the law of $\pi_{N}$ when $\zeta$ is distributed according to $\nu_{N}^{0}$ which is the uniform probability on $\Omega_{N}$.

Lemma 3.2. The sequence $\left\{\mathcal{P}_{N}^{0}\right\}$ satisfies a large deviation principle with rate function $\mathcal{S}$. Namely, for each closed set $\mathcal{C} \subset \mathcal{M}$ and each open set $\mathcal{O} \subset \mathcal{M}$

$$
\begin{aligned}
& \varlimsup_{N \rightarrow \infty} \frac{1}{N} \log \mathcal{P}_{N}^{0}(\mathcal{C}) \leq-\inf _{\rho \in \mathcal{C}} \mathcal{S}(\rho) \\
& \varliminf_{N \rightarrow \infty} \frac{1}{N} \log \mathcal{P}_{N}^{0}(\mathcal{O}) \geq-\inf _{\rho \in \mathcal{O}} \mathcal{S}(\rho) .
\end{aligned}
$$

Proof. The proof is split in few steps.

Step 1. Set

$$
\mathcal{A}:=\left\{\lambda \in C^{1}\left(\mathbf{T} ; \mathbf{R}^{3}\right): \int_{0}^{1} d r \frac{e^{\lambda_{\alpha}(r)}}{e^{\lambda_{A}(r)}+e^{\lambda_{B}(r)}+e^{\lambda_{C}(r)}}=\frac{1}{3}, \alpha \in\{A, B, C\}\right\}
$$

and let $\Lambda: \mathcal{A} \rightarrow \mathbf{R}$ be the functional

$$
\Lambda(\lambda):=\int_{0}^{1} d r \log \left[\frac{1}{3}\left(e^{\lambda_{A}(r)}+e^{\lambda_{B}(r)}+e^{\lambda_{C}(r)}\right)\right]
$$

we shall prove that for each $\lambda \in \mathcal{A}$

$$
\lim _{N \rightarrow \infty} \frac{1}{N} \log \int d \mathcal{P}_{N}^{0}(\rho) \exp \{N\langle\lambda, \rho\rangle\}=\Lambda(\lambda) .
$$

Denote by $\lambda^{N}(x)$ the average of $\lambda$ in the interval $[x / N,(x+1) / N)$,

$$
\lambda_{\alpha}^{N}(x):=N \int_{\frac{x}{N}}^{\frac{x+1}{N}} d r \lambda_{\alpha}(r), \quad x \in \mathbf{Z}_{N}, \alpha \in\{A, B, C\} .
$$

From the very definition of the measure $\mathcal{P}_{N}^{0}$,

$$
\begin{aligned}
\int d \mathcal{P}_{N}^{0}(\rho) \exp \{N\langle\lambda, \rho\rangle\} & =\sum_{\zeta \in \Omega_{N}} \nu_{N}^{0}(\zeta) \exp \left\{N\left\langle\lambda, \pi^{N}(\zeta)\right\rangle\right\} \\
& =\sum_{\zeta \in \Omega_{N}} \nu_{N}^{0}(\zeta) \prod_{x \in \mathbf{Z}_{N}} \exp \left\{\sum_{\alpha \in\{A, B, C\}} \lambda_{\alpha}^{N}(x) \eta_{\alpha}(x)\right\}
\end{aligned}
$$


We denote by $\mu_{N}^{\lambda}$ the product measure on $\widetilde{\Omega}_{N}=\{A, B, C\}^{\mathbf{Z}_{N}}$ with marginals $\mu_{N, x}^{\lambda}$ given by

$$
\mu_{N, x}^{\lambda}(\alpha)=\frac{e^{\lambda_{\alpha}^{N}(x)}}{e^{\lambda_{A}^{N}(x)}+e^{\lambda_{B}^{N}(x)}+e^{\lambda_{C}^{N}(x)}}, \quad \alpha \in\{A, B, C\} .
$$

When $\lambda=0$ we drop the superscript $\lambda$ from the notation so that $\mu_{N}$ is the uniform measure on $\widetilde{\Omega}_{N}$.

Set $\Xi_{N}(\lambda):=\prod_{x \in \mathbf{Z}_{N}}\left(e^{\lambda_{A}^{N}(x)}+e^{\lambda_{B}^{N}(x)}+e^{\lambda_{C}^{N}(x)}\right)$. As $\nu_{N}^{0}=\mu_{N}\left(\cdot \mid \Omega_{N}\right)$, from (3.11) we get

$$
\int d \mathcal{P}_{N}^{0}(\rho) \exp \{N\langle\lambda, \rho\rangle\}=\frac{\Xi_{N}(\lambda)}{3^{N}} \frac{\mu_{N}^{\lambda}\left(\Omega_{N}\right)}{\mu_{N}\left(\Omega_{N}\right)} .
$$

We claim that for each $\lambda \in \mathcal{A}$ it holds $\lim _{N} \frac{1}{N} \log \mu_{N}^{\lambda}\left(\Omega_{N}\right)=0$. The proof of this step is then completed by observing that $\frac{1}{N} \log \left[\Xi_{N}(\lambda) / 3^{N}\right] \rightarrow \Lambda(\lambda)$.

To prove the claim, we write

$$
\mu_{N}^{\lambda}\left(\Omega_{N}\right)=\mu_{N}^{\lambda}\left(\frac{1}{N} \sum_{x \in \mathbf{Z}_{N}} \eta_{\alpha}(x)=\frac{1}{3}, \alpha \in\{A, B, C\}\right) .
$$

In view of the smoothness of $\lambda$ and the constraints in (3.8), for each $\alpha \in\{A, B, C\}$

$$
\begin{aligned}
\frac{1}{N} \sum_{x \in \mathbf{Z}_{N}} \mu_{N}^{\lambda}\left(\eta_{\alpha}(x)\right) & =\frac{1}{N} \sum_{x \in \mathbf{Z}_{N}} \frac{e^{\lambda_{\alpha}^{N}(x)}}{e^{\lambda_{A}^{N}(x)}+e^{\lambda_{B}^{N}(x)}+e^{\lambda_{C}^{N}(x)}} \\
& =\int_{0}^{1} d r \frac{e^{\lambda_{\alpha}(r)}}{e^{\lambda_{A}(r)}+e^{\lambda_{B}(r)}+e^{\lambda_{C}(r)}}+O\left(\frac{1}{N}\right)=\frac{1}{3}+O\left(\frac{1}{N}\right) .
\end{aligned}
$$

The claim now follows from an application of the local central limit theorem for triangular arrays, see e.g. [23, Ch. VII].

Step 2. We here prove the large deviations upper bound (3.6). Given $\lambda \in \mathcal{A}$, let $\mathcal{P}_{N}^{0, \lambda}$ be the probability on $\mathcal{M}$ defined by

$$
d \mathcal{P}_{N}^{0, \lambda}:=\exp \left\{N\left[\langle\lambda, \cdot\rangle-\Lambda_{N}(\lambda)\right]\right\} d \mathcal{P}_{N}^{0}
$$

where

$$
\Lambda_{N}(\lambda)=\frac{1}{N} \log \int d \mathcal{P}_{N}^{0}(\rho) e^{N\langle\lambda, \rho\rangle} .
$$

Given a measurable subset $\mathcal{B}$ of $\mathcal{M}$, we then have

$$
\mathcal{P}_{N}^{0}(\mathcal{B})=\int_{\mathcal{B}} d \mathcal{P}_{N}^{0, \lambda} \frac{d \mathcal{P}_{N}^{0}}{d \mathcal{P}_{N}^{0, \lambda}} \leq \sup _{\rho \in \mathcal{B}} \exp \left\{-N\left[\langle\lambda, \rho\rangle-\Lambda_{N}(\lambda)\right]\right\} .
$$

In view of Step $1, \Lambda_{N}(\lambda) \rightarrow \Lambda(\lambda)$ as $N \rightarrow \infty$. We thus get

$$
\varlimsup_{N \rightarrow \infty} \frac{1}{N} \log \mathcal{P}_{N}^{0}(\mathcal{B}) \leq-\inf _{\rho \in \mathcal{B}}\{\langle\lambda, \rho\rangle-\Lambda(\lambda)\} .
$$

By optimizing with respect to $\lambda \in \mathcal{A}$ and using a mini-max lemma, see e.g. Lemmata 3.2 and 3.3 in [20, App. 2], we deduce that for each compact $\mathcal{K} \subset \mathcal{M}$

$$
\varlimsup_{N \rightarrow \infty} \frac{1}{N} \log \mathcal{P}_{N}^{0}(\mathcal{K}) \leq-\inf _{\rho \in \mathcal{K}} \sup _{\lambda \in \mathcal{A}}\{\langle\lambda, \rho\rangle-\Lambda(\lambda)\}=-\inf _{\rho \in \mathcal{K}} \mathcal{S}(\rho)
$$

where the last identity follows by Legendre duality. By the compactness of $\mathcal{M}$ this concludes the proof of the upper bound.

Step 3. Given two probability measures $P$ and $Q$, we denote by $\operatorname{Ent}(Q \mid P)=$ $\int d Q \log [d Q / d P]$ the relative entropy of $Q$ with respect to $P$. A simple computation 
based on Jensen inequality, see e.g. [19, Prop. 4.1], shows that the large deviations lower bound (3.7) can be deduced from the following statement. For each $\rho \in \mathcal{M}$ there exists a sequence of probability measures $\left\{\mathcal{Q}_{N}^{\rho}\right\}$ such that

$$
\mathcal{Q}_{N}^{\rho} \rightarrow \delta_{\rho} \quad \text { and } \quad \varlimsup_{N \rightarrow \infty} \frac{1}{N} \operatorname{Ent}\left(\mathcal{Q}_{N}^{\rho} \mid \mathcal{P}_{N}^{0}\right) \leq \mathcal{S}(\rho)
$$

We here construct the sequence $\left\{\mathcal{Q}_{N}^{\rho}\right\}$ when $\rho$ is continuously differentiable and bounded away from 0 and 1 . For such a $\rho$ let $\lambda=\lambda(\rho)$ be such that

$$
\rho_{\alpha}=\frac{e^{\lambda_{\alpha}}}{e^{\lambda_{A}}+e^{\lambda_{B}}+e^{\lambda_{C}}}, \quad \alpha \in\{A, B, C\} .
$$

Observe that $\lambda \in \mathcal{A}$ since $\rho$ is continuously differentiable and bounded away from 0 and 1. Recalling (3.12), we claim that $\left\{\mathcal{P}_{N}^{0, \lambda(\rho)}\right\}$ fulfils the condition (3.13). The law of large numbers $\mathcal{P}_{N}^{0, \lambda(\rho)} \rightarrow \delta_{\rho}$ can be indeed checked by the same computations of Step 1. Furthermore, in view of such law of large numbers and Step 1,

$$
\lim _{N \rightarrow \infty} \frac{1}{N} \operatorname{Ent}\left(\mathcal{P}_{N}^{0, \lambda(\rho)} \mid \mathcal{P}_{N}^{0}\right)=\langle\lambda, \rho\rangle-\Lambda(\lambda)=\mathcal{S}(\rho)
$$

where the last equality follows from the choice of $\lambda$ and Legendre duality.

Step 4. The proof of the lower bound is here concluded by an approximation argument [19, Prop. 4.1]. Let $\mathcal{M}$ 。 be the subset of $\mathcal{M}$ given by the continuously differentiable profiles bounded away from 0 and 1 . The condition that a large deviation rate function is lower semicontinuous is not restrictive. More precisely, if a sequence of probabilities satisfies the large deviations lower bound for some rate function, then the lower bound still holds with the lower semicontinuous envelope of such rate function. If we let $\mathcal{S}^{\circ}$ be the functional equal to $\mathcal{S}$ on $\mathcal{M}_{\circ}$ and $\mathcal{S}^{\circ}(\rho)=+\infty$ otherwise, in view of Step 3, the proof of the lower bound (3.7) is concluded if we show that the lower semicontinuous envelope of $\mathcal{S}^{\circ}$ is $\mathcal{S}$. This amounts to prove that

$$
\mathcal{S}(\rho)=\sup _{\mathcal{O} \ni \rho} \inf _{\hat{\rho} \in \mathcal{O} \cap \mathcal{M}_{\circ}} \mathcal{S}(\hat{\rho})
$$

where the first supremum is carried over all the open neighborhoods of $\rho$. The previous identity is easily proven by considering a sequence $\left\{\rho^{n}\right\}$ of continuously differentiable profiles bounded away from 0 and 1 which converges to $\rho$ a.e. in $\mathbf{T}$.

In view of the continuity of the functional $\mathcal{H}$ on $\mathcal{M}$, the large deviations principle for the sequence $\left\{\mathcal{P}_{N}^{\beta}\right\}$ is straightforward consequence of Lemma 3.2 and LaplaceVaradhan theorem.

Proof of Theorem 3.1. Recalling definitions (2.5), (3.2) and (3.4), we claim that for each $\zeta \in \Omega_{N}$

$$
H_{N}(\zeta)=\mathcal{H}\left(\pi^{N}(\zeta)\right)
$$

It is indeed enough to notice that by writing explicitly the right hand side above the diagonal terms vanish since $\eta_{A}(x)+\eta_{B}(x)+\eta_{C}(x)=1, x \in \mathbf{Z}_{N}$. 
Recall that $\nu_{N}^{0}$ is the uniform probability on $\Omega_{N}$ and let $\mathcal{B}$ be a measurable subset of $\mathcal{M}$. From (3.14) and the definitions of the measures $\mathcal{P}_{N}^{\beta}$ and $\nu_{N}^{\beta}$, see (2.6)

$$
\begin{aligned}
\mathcal{P}_{N}^{\beta}(\mathcal{B}) & =\sum_{\substack{\zeta \in \Omega_{N} \\
\pi^{N}(\zeta) \in \mathcal{B}}} \nu_{N}^{\beta}(\zeta)=\frac{\left|\Omega_{N}\right|}{Z_{N}^{\beta}} \sum_{\substack{\zeta \in \Omega_{N} \\
\pi^{N}(\zeta) \in \mathcal{B}}} \nu_{N}^{0}(\zeta) e^{-\beta N H_{N}(\zeta)} \\
& =\frac{\left|\Omega_{N}\right|}{Z_{N}^{\beta}} \int_{\mathcal{B}} d \mathcal{P}_{N}^{0} e^{-\beta N \mathcal{H}\left(\pi_{N}\right)} .
\end{aligned}
$$

In particular, by taking $\mathcal{B}=\mathcal{M}$,

$$
\frac{Z_{N}^{\beta}}{\left|\Omega_{N}\right|}=\int d \mathcal{P}_{N}^{0} e^{-\beta N \mathcal{H}\left(\pi_{N}\right)}
$$

Since $\mathcal{M}$ is compact and $\mathcal{H}: \mathcal{M} \rightarrow \mathbf{R}$ is continuous, by using Lemma 3.2 and Laplace-Varadhan theorem, see e.g. [10, Thm. 4.3.1], we deduce

$$
\lim _{N \rightarrow \infty} \frac{1}{N} \log \frac{Z_{N}^{\beta}}{\left|\Omega_{N}\right|}=\sup _{\rho \in \mathcal{M}}\{-\beta \mathcal{H}(\rho)-\mathcal{S}(\rho)\}=-\inf _{\rho \in \mathcal{M}} \mathcal{F}_{\beta}(\rho)
$$

Let $\mathcal{C}$ and $\mathcal{O}$ be respectively a closed and an open subset of $\mathcal{M}$. Again from Lemma 3.2 and Laplace-Varadhan theorem, see e.g. [10, Ex. 4.3.11], we deduce

$$
\begin{aligned}
\varlimsup_{N \rightarrow \infty} \frac{1}{N} \log \int_{\mathcal{C}} d \mathcal{P}_{N}^{0} e^{-\beta N \mathcal{H}\left(\pi^{N}\right)} & \leq-\inf _{\rho \in \mathcal{C}} \mathcal{F}_{\beta}(\rho) \\
\varliminf_{N \rightarrow \infty} \frac{1}{N} \log \int_{\mathcal{O}} d \mathcal{P}_{N}^{0} e^{-\beta N \mathcal{H}\left(\pi^{N}\right)} & \geq-\inf _{\rho \in \mathcal{O}} \mathcal{F}_{\beta}(\rho) .
\end{aligned}
$$

The theorem follows readily.

Minimizers of the free energy. We here recall the results in [1] concerning the minimizers of the free energy $\mathcal{F}_{\beta}$ in (3.5) that are needed in our analysis. As discussed in [1], the Euler-Lagrange equation $\delta \mathcal{F}_{\beta}=0$ can be, equivalently, written as the system of ordinary differential equations

$$
\begin{aligned}
& \rho_{A}^{\prime}=\beta \rho_{A}\left(\rho_{C}-\rho_{B}\right) \\
& \rho_{B}^{\prime}=\beta \rho_{B}\left(\rho_{A}-\rho_{C}\right) \\
& \rho_{C}^{\prime}=\beta \rho_{C}\left(\rho_{B}-\rho_{A}\right) .
\end{aligned}
$$

Note that the above condition is equivalent to the statement that $\rho=\left(\rho_{A}, \rho_{B}, \rho_{C}\right)$ is a stationary solution to the hydrodynamic equation (1.1).

Let $\beta_{\mathrm{c}}:=2 \pi \sqrt{3}$. In [1] it is proven that for $\beta \in\left[0, \beta_{\mathrm{c}}\right]$ the unique solution to (3.15) in $\mathcal{M}$ is the homogeneous profile $\bar{\rho}:=\left(\frac{1}{3}, \frac{1}{3}, \frac{1}{3}\right)$. On the other hand, when $\beta>\beta_{\mathrm{c}}$ there are non trivial solutions. In particular, there exists a unique $\rho \in C^{\infty}\left(\mathbf{R} ; \mathbf{R}^{3}\right)$ satisfying the following conditions: (i) $\rho$ solves (3.15), (ii) $\rho$ is periodic with period 1, (iii) $\rho$ satisfies the constraints in (3.1) and can therefore be thought as an element in $\mathcal{M}$, (iv) the center of mass of the $B$ species is $1 / 2$, i.e. $3 \int_{0}^{1} d r r \rho_{B}(r)=1 / 2$. We shall denote this solution by $\rho^{*}=\rho^{*, \beta}$. Note that any translation of $\rho^{*, \beta}$ satisfies conditions (i)-(iii) above but not (iv). We emphasize that the condition (ii) requires the minimal period to be one. Indeed, as discussed in [1, when $\beta>n \beta_{\mathrm{c}}$ for some integer $n \geq 2$, there are solutions of (3.15) with period $1 / n$. These are the other critical points of $\mathcal{F}_{\beta}$ which may lead to a metastable behavior. 
Given $s \in \mathbf{T}$ we denote by $\tau_{s}: \mathcal{M} \rightarrow \mathcal{M}$ the translation by $s$, namely $\left(\tau_{s} \rho\right)(r)=$ $\rho(r-s)$. If $\mathcal{P}$ is a probability on $\mathcal{M}$, the corresponding translation is $\mathcal{P} \circ \tau_{s}^{-1}$. The following statement is a (partial) rewriting of Theorems 4.1 and 5.2 in [1].

\section{Theorem 3.3.}

(i) If $\beta \in\left[0, \beta_{\mathrm{c}}\right]$ then

$$
\arg \inf \mathcal{F}_{\beta}=\{\bar{\rho}\}
$$

namely, the unique minimizer of $\mathcal{F}_{\beta}$ is $\bar{\rho}$.

(ii) If $\beta \in\left(\beta_{\mathrm{c}},+\infty\right)$ then

$$
\arg \inf \mathcal{F}_{\beta}=\left\{\tau_{s} \rho^{*, \beta}, s \in \mathbf{T}\right\}
$$

namely, $\mathcal{F}_{\beta}$ has a one-parameter family of minimizers which are obtained by translating $\rho^{*, \beta}$.

Law of large numbers for the empirical density. As a corollary of the previous statements, we here prove the law of large numbers for the sequence $\left\{\mathcal{P}_{N}^{\beta}\right\}$. The corresponding limit point charges the set of minimizers of the free energy only. In the supercritical case we show that each $\tau_{s} \rho^{*, \beta}, s \in \mathbf{T}$, is chosen with uniform probability.

\section{Theorem 3.4.}

(i) If $\beta \in\left[0, \beta_{\mathrm{c}}\right]$ then the sequence $\left\{\mathcal{P}_{N}^{\beta}\right\}$ converges to $\delta_{\bar{\rho}}$.

(ii) If $\beta \in\left(\beta_{\mathrm{c}},+\infty\right)$ then the sequence $\left\{\mathcal{P}_{N}^{\beta}\right\}$ converges to $\int_{0}^{1} d s \delta_{\tau_{s} \rho^{*, \beta}}$.

Proof. Item (i) follows immediately from the large deviations principle stated in Theorem 3.1 and the uniqueness of minimizers of $\mathcal{F}_{\beta}$ stated in item (i) of Theorem 3.3 ,

To prove item (ii), let $\vartheta: \Omega_{N} \rightarrow \Omega_{N}$ be the microscopic translation, i.e. $\vartheta \zeta$ is the configuration defined by $(\vartheta \zeta)(x)=\zeta(x-1), x \in \mathbf{Z}_{N}$. As follows from definition (2.6), the probability $\nu_{N}^{\beta}$ is translation invariant, i.e. $\nu_{N}^{\beta} \circ \vartheta^{-1}=\nu_{N}^{\beta}$. This implies that the probability $\mathcal{P}_{N}^{\beta}$ is invariant by discrete translations: $\mathcal{P}_{N}^{\beta} \circ \tau_{x / N}^{-1}=\mathcal{P}_{N}^{\beta}$, $x \in \mathbf{Z}_{N}$. By the compactness of $\mathcal{M}$, there exists a probability $\mathcal{P} \in \mathcal{P}(\mathcal{M})$ and a subsequence $\left\{\mathcal{P}_{N}^{\beta}\right\}$ such that $\mathcal{P}_{N}^{\beta} \rightarrow \mathcal{P}$. We claim that $\mathcal{P}$ is translation invariant. Indeed, fix a continuous function $F$ on $\mathcal{M}$ and $s \in \mathbf{T}$. Observe that, in view of the compactness of $\mathcal{M}, F$ is uniformly continuous. Pick now a sequence $\left\{x_{N} \in \mathbf{Z}_{N}\right\}$ such that $x_{N} / N \rightarrow s$. The uniform continuity of $F$ implies that $\tau_{x_{N} / N} F$ converges uniformly to $\tau_{s} F$. Since $\int d \mathcal{P}_{N}^{\beta} \tau_{x_{N} / N} F=\int d \mathcal{P}_{N}^{\beta} F$, by taking the limit $N \rightarrow \infty$ we deduce that $\int d \mathcal{P} \tau_{s} F=\int d \mathcal{P} F$. In view of the arbitrariness of $F$ we conclude $\mathcal{P} \circ \tau_{s}^{-1}=\mathcal{P}$. Moreover, Theorem 3.1 and item (ii) in Theorem 3.3 imply that the support of $\mathcal{P}$ is a subset of $\left\{\tau_{s} \rho^{*, \beta}, s \in \mathbf{T}\right\}=: \mathcal{T}$. Let now $\phi: \mathcal{T} \rightarrow \mathbf{T}$ be the bijection defined by $\tau_{s} \rho^{*, \beta} \mapsto s$ and set $\lambda:=\mathcal{P} \circ \phi^{-1}$. Since $\mathcal{P}=\mathcal{P} \circ \tau_{s}^{-1}, s \in \mathbf{T}$, we deduce that $\lambda$ is a translation invariant probability measure on $\mathbf{T}$. As the Lebesgue measure $d r$ is the unique translation invariant probability measure on $\mathbf{T}$ we deduce $\lambda(d r)=d r$. The proof is now completed by observing that for each continuous $F: \mathcal{M} \rightarrow \mathbf{R}$ the previous identity imply $\int d \mathcal{P}(\rho) F(\rho)=\int_{0}^{1} d s F\left(\tau_{s} \rho^{*}\right)$.

\section{LOWER BOUND ON THE SPECTRAL GAP IN THE SUBCRITICAL CASE}

In this section we prove the first statement in Theorem 2.1. This result is derived from an analysis of a perturbed interchange process, that is detailed in Appendix $\mathrm{A}$ 
and a comparison of the corresponding Dirichlet forms. This method has been introduced in 24] and applied in different contexts, see e.g. [5].

We start by defining the $\mathrm{ABC}$ process on the complete graph with $N$ vertices. Given a (unoriented) bond $\{x, y\} \subset \mathbf{Z}_{N}, x \neq y$, and a function $f: \Omega_{N} \rightarrow \mathbf{R}$, we introduce the gradient

$$
\left(\nabla_{x, y} f\right)(\zeta):=f\left(\zeta^{x, y}\right)-f(\zeta)
$$

where, as in (2.1), $\zeta^{x, y}$ denotes the configuration obtained from $\zeta$ exchanging the particles in $x$ and $y$. The ABC dynamics on the complete graph is then defined by the Markov generator

$$
\mathcal{L}_{N}^{\beta} f:=\sum_{\{x, y\} \subset \mathbf{Z}_{N}} c_{x, y}^{\beta} \nabla_{x, y} f
$$

where, recalling (2.5), the jump rates $c_{x, y}^{\beta}=c_{x, y}^{\beta, N}: \Omega_{N} \rightarrow(0,+\infty)$ are given by

$$
c_{x, y}^{\beta, N}:=\frac{1}{N} \exp \left\{-\frac{\beta N}{2} \nabla_{x, y} H_{N}\right\} .
$$

In particular, the above rates satisfy the detailed balance with respect to the probability measure $\nu_{N}^{\beta}$ defined in (2.6). Recalling (2.3) and (2.5), we also observe that $N c_{x, x+1}^{\beta, N}=c_{x}^{\beta, N}$.

In Appendix $\AA$ we prove that, provided $\beta$ is small enough, the spectral gap of $\mathcal{L}_{N}^{\beta}$ is of order one uniformly in $N$.

Lemma 4.1. There exist constants $\beta_{0}, C_{1} \in(0,+\infty)$ such that for any $\beta \in\left[0, \beta_{0}\right]$ and any $N \geq 3$

$$
\operatorname{gap}\left(\mathcal{L}_{N}^{\beta}\right) \geq \frac{1}{C_{1}}
$$

We denote by $D_{N}^{\beta}$ and $\mathcal{D}_{N}^{\beta}$ the Dirichlet forms associated to the generators $L_{N}^{\beta}$ and $\mathcal{L}_{N}^{\beta}$, respectively. That is, given $f: \Omega_{N} \rightarrow \mathbf{R}$,

$$
\begin{aligned}
& D_{N}^{\beta}(f, f):=\nu_{N}^{\beta}\left(f\left(-L_{N}^{\beta}\right) f\right)=\frac{1}{2} \sum_{x=1}^{N} \nu_{N}^{\beta}\left(c_{x}^{\beta}\left[\nabla_{x, x+1} f\right]^{2}\right), \\
& \mathcal{D}_{N}^{\beta}(f, f):=\nu_{N}^{\beta}\left(f\left(-\mathcal{L}_{N}^{\beta}\right) f\right)=\frac{1}{2} \sum_{\{x, y\} \subset \mathbf{Z}_{N}} \nu_{N}^{\beta}\left(c_{x, y}^{\beta}\left[\nabla_{x, y} f\right]^{2}\right) .
\end{aligned}
$$

Lemma 4.2. The inequality

$$
\mathcal{D}_{N}^{\beta}(f, f) \leq 2 e^{3 \beta} N^{2} D_{N}^{\beta}(f, f)
$$

holds for any $\beta \geq 0$ and any function $f: \Omega_{N} \rightarrow \mathbf{R}$.

Proof. Given $\{x, y\} \subset \mathbf{Z}_{N}$ we let $T_{x, y}: \Omega_{N} \rightarrow \Omega_{N}$ be the involution defined by $T_{x, y} \zeta:=\zeta^{x, y}$. We use the same notation for the corresponding linear map on the set of functions $f: \Omega_{N} \rightarrow \mathbf{R}$, i.e. $\left(T_{x, y} f\right)(\zeta):=f\left(T_{x, y} \zeta\right)=f\left(\zeta^{x, y}\right)$. As it is simple to check, the long jump $T_{x, y}$ can be decomposed in terms of nearest neighbor jumps as follows

$$
T_{x, y}=T_{x+1, x} T_{x+2, x+1} \cdots T_{y-1, y-2} T_{y-1, y} T_{y-2, y-1} \cdots T_{x+1, x+2} T_{x, x+1} .
$$


We then write the telescopic sum

$$
\begin{aligned}
& T_{x, y} f-f \\
& \quad=\left[T_{x, x+1} \cdots T_{y, y-1} \cdots T_{x+2, x+1} T_{x+1, x} f-T_{x, x+1} \cdots T_{y, y-1} \cdots T_{x+2, x+1} f\right] \\
& \quad+\left[T_{x, x+1} \cdots T_{y, y-1} \cdots T_{x+3, x+2} T_{x+2, x+1} f-T_{x, x+1} \cdots T_{y, y-1} \cdots T_{x+3, x+2} f\right] \\
& \quad+\cdots+\left[T_{x, x+1} \cdots T_{y-2, y-1} T_{y, y-1} f-T_{x, x+1} \cdots T_{y-2, y-1} f\right] \\
& \quad+\cdots+\left[T_{x, x+1} f-f\right] .
\end{aligned}
$$

Whence

$$
\begin{aligned}
& \nabla_{x, y} f(\zeta)=\left(\nabla_{x, x+1} f\right)\left(T_{x+1, x} \cdots T_{y-1, y} \cdots T_{x+1, x+2} \zeta\right) \\
& \quad+\left(\nabla_{x+1, x+2} f\right)\left(T_{x+1, x} \cdots T_{y-1, y} \cdots T_{x+2, x+3} \zeta\right) \\
& \quad+\cdots+\left(\nabla_{y-1, y} f\right)\left(T_{x+1, x} \cdots T_{y-2, y-1} \zeta\right)+\cdots+\left(\nabla_{x, x+1} f\right)(\zeta)
\end{aligned}
$$

In view of (2.5) and (2.6), for any $\beta \in \mathbf{R}_{+}$, any $z \in \mathbf{Z}_{N}$, and any positive function $g: \Omega_{N} \rightarrow \mathbf{R}_{+}$

$$
\nu_{N}^{\beta}\left(T_{z, z+1} g\right) \leq \exp \left\{N \beta\left\|\nabla_{z, z+1} H_{N}\right\|_{\infty}\right\} \nu_{N}^{\beta}(g) \leq e^{\beta / N} \nu_{N}^{\beta}(g)
$$

where $\|\cdot\|_{\infty}$ denotes the uniform norm. By Schwarz inequality in (4.6) and using recursively the previous estimate we then get, for $1 \leq x<y \leq N$

$$
\nu_{N}^{\beta}\left(\left[\nabla_{x, y} f\right]^{2}\right) \leq 2[2(y-x)-1] e^{2 \beta} \sum_{z=x}^{y-1} \nu_{N}^{\beta}\left(\left[\nabla_{z, z+1} f\right]^{2}\right) .
$$

Indeed, in the generic term on the right hand side of (4.6) there is the composition of nearest neighbor exchanges $T_{x+k, x+k+1}$ whose number is at most $2(y-x)-2 \leq 2 N$. In view of (4.7) this yields the factor $e^{2 \beta}$. As the number of terms on the right hand side of (4.6) is $2(y-x)-1$ and each bond $\{z, z+1\}, z=x, \cdots, y-1$ is used at most two times, the bound (4.8) follows.

To conclude the proof of the lemma it is now enough to observe that the jump rates in the Dirichlet forms (4.4) and (4.5) respectively satisfy the bounds $c_{x}^{\beta} \geq$ $e^{-\beta /(2 N)}$ and $N c_{x, y}^{\beta} \leq e^{\beta / 2}$. In view of (4.8) elementary computations now yield the statement.

Proof of Theorem [2.1, item (i). Recall the Rayleigh-Ritz variational characterization of the spectral gap (2.7). By Lemmata 4.1 and 4.2 we then deduce the statement with $\beta_{0}$ as in Lemma 4.1 and $C_{0}=\frac{1}{2} e^{-3 \beta_{0}} C_{1}$.

\section{UPPER BOUND ON THE SPECTRAL GAP IN THE SUPERCRITICAL CASE}

We discuss here the upper bound on the spectral gap when $\beta>\beta_{\mathrm{c}}$. In view of the Rayleigh-Ritz variational characterization (2.7), the proof will be achieved by exhibiting a suitable test function. The naive picture is the following. When $\beta>\beta_{\mathrm{c}}$ and $N$ is large, the $\mathrm{ABC}$ process essentially performs a random walk on the set of minimizers of the free energy $\mathcal{F}_{\beta}$, which in the supercritical case is homeomorphic to the one-dimensional torus. We thus choose as test function the one that corresponds to the slow mode of such random walk and conclude the argument. 
Proof of Theorem 2.1, item (ii). Pick a Lipschitz function $\phi: \mathbf{T} \rightarrow \mathbf{R}$ such that $\int_{0}^{1} d r \phi(r)=0$ to be chosen later and let $f_{N}: \Omega_{N} \rightarrow \mathbf{R}$ be the function

$$
f_{N}:=\frac{1}{N} \sum_{x=1}^{N} \eta_{B}(x) \phi\left(\frac{x}{N}\right) .
$$

By the Rayleigh-Ritz principle,

$$
\operatorname{gap}\left(L_{N}^{\beta}\right) \leq \frac{D_{N}^{\beta}\left(f_{N}, f_{N}\right)}{\nu_{N}^{\beta}\left(f_{N}, f_{N}\right)}
$$

where the Dirichlet form $D_{N}^{\beta}$ has been defined in (4.4). We next estimate from below the denominator and from above the numerator.

To bound the variance of $f_{N}$, we first observe that, since $\phi$ has mean zero, we have $\lim _{N} \nu_{N}^{\beta}\left(f_{N}\right)=\frac{1}{3} \int_{0}^{1} d r \phi(r)=0$. Recall (3.1) and let $F: \mathcal{M} \rightarrow \mathbf{R}$ be defined by

$$
F(\rho)=\int_{0}^{1} d r \rho_{B}(r) \phi(r)
$$

The continuity of $\phi$ implies

$$
\lim _{N \rightarrow \infty} \sup _{\zeta \in \Omega_{N}}\left|f_{N}(\zeta)-F\left(\pi_{N}(\zeta)\right)\right|=0,
$$

where the empirical density $\pi_{N}: \Omega_{N} \rightarrow \mathcal{M}$ has been defined in (3.2). By assumption, $\beta>\beta_{\mathrm{c}}$ and therefore Theorem 3.4 , item (ii) implies

$$
\lim _{N \rightarrow \infty} \nu_{N}^{\beta}\left(f_{N}^{2}\right)=\lim _{N \rightarrow \infty} \nu_{N}^{\beta}\left(F\left(\pi_{N}\right)^{2}\right)=\int_{0}^{1} d s\left[\int_{0}^{1} d r \rho_{B}^{*, \beta}(r-s) \phi(r)\right]^{2} .
$$

We can choose $\phi$ such that the right hand side above is strictly positive. It is indeed enough to observe that, since $\rho^{*, \beta}$ is not constant, there exists a Lipschitz, mean zero, function $\phi$ such that $\int_{0}^{1} d r \rho_{B}^{*, \beta}(r) \phi(r) \neq 0$. For such choice of $\phi$ we deduce there exists a constant $C(\beta) \in(0,+\infty)$ such that $\nu_{N}^{\beta}\left(f_{N}, f_{N}\right) \geq C(\beta)$ for any $N \geq 3$.

We next bound the Dirichlet form. A straightforward computation yields

$$
\nabla_{x, x+1} f_{N}=\frac{1}{N}\left[\phi\left(\frac{x+1}{N}\right)-\phi\left(\frac{x}{N}\right)\right]\left[\eta_{B}(x)-\eta_{B}(x+1)\right] .
$$

Since $c_{x}^{\beta, N} \leq \exp \left\{\frac{\beta}{2 N}\right\}$, we then get

$$
D_{N}^{\beta}\left(f_{N}, f_{N}\right) \leq \exp \left\{\frac{\beta}{2 N}\right\} \frac{1}{2 N^{2}} \sum_{x=1}^{N}\left[\phi\left(\frac{x+1}{N}\right)-\phi\left(\frac{x}{N}\right)\right]^{2} .
$$

Therefore, letting $C_{\phi}$ be the Lipschitz constant of $\phi$,

$$
\varlimsup_{N \rightarrow \infty} N^{3} D_{N}^{\beta}\left(f_{N}, f_{N}\right) \leq \frac{1}{2} C_{\phi}^{2}
$$

which concludes the proof. 


\section{Appendix A. Spectral gap for Perturbed interchange Processes}

We prove here a general result on the spectral gap on suitable Markov chains on the set of permutations of $\{1, \ldots, N\}$. The jumps of this chain are obtained by randomly choosing a transposition. As reference process we consider the so-called interchange process on $\{1, \ldots, N\}$, see [8, 11]. This process can be realized as the simple random walk on the graph with vertex set given by the symmetric group $S_{N}$ and edges given by the pairs $\left(\sigma_{1}, \sigma_{2}\right) \in S_{N} \times S_{N}$ such that the composition $\sigma_{1}^{-1} \circ \sigma_{2}$ is a transposition. The reference invariant measure is thus the uniform probability on the symmetric group. We then perturb this measure according to the standard Gibbs formalism and consider an associated reversible chain. Under general conditions on the energy, we show that - at high enough temperature - the relaxation time of perturbed chain behaves, for large $N$, as the one of the reference random walk. The $\mathrm{ABC}$ dynamics on the complete graph (4.2) can be realized by looking at the previous chain in a colorblind way, that is resolving only 3 out of the $N$ colors.

Let $V_{N}:=\{1, \ldots, N\}$ and $B_{N}:=\left\{b \subset V_{N}:|b|=2\right\}$. The complete graph on $N$ vertices is $G_{N}:=\left(V_{N}, B_{N}\right)$ and $S_{N}:=\left\{\sigma: V_{N} \rightarrow V_{N}\right.$, bijective $\}$ is the set of permutations on $V_{N}$. For $\sigma \in S_{N},\{x, y\} \in B_{N}$ let $\sigma^{\{x, y\}} \in S_{N}$ be the permutation obtained by composing $\sigma$ with the transposition which exchanges $x$ and $y$

$$
\sigma^{\{x, y\}}(z):= \begin{cases}\sigma(y) & \text { if } z=x \\ \sigma(x) & \text { if } z=y \\ \sigma(z) & \text { otherwise. }\end{cases}
$$

Given an energy function $E_{N}: S_{N} \rightarrow \mathbf{R}$ and $\beta \geq 0$, we define the probability on $S_{N}$ by

$$
\pi_{N}(\sigma)=\pi_{N}^{\beta}(\sigma):=\frac{1}{Z_{N}^{\beta}} \exp \left\{-\beta E_{N}(\sigma)\right\}
$$

where $Z_{N}^{\beta}$ is the normalization constant. For $f: S_{N} \rightarrow \mathbf{R}, a \in B_{N}$ define $f^{a}(\sigma):=$ $f\left(\sigma^{a}\right), \nabla_{a} f:=f^{a}-f$ and the Markov generator $\mathcal{G}_{N}=\mathcal{G}_{N}^{\beta}$ by

$$
\mathcal{G}_{N} f:=\sum_{a \in B_{N}} c_{a} \nabla_{a} f
$$

where the transition rates are

$$
c_{a}=c_{a}^{\beta, N}:=\frac{1}{N} \exp \left\{-\frac{\beta}{2} \nabla_{a} E_{N}\right\} .
$$

The associated Markov chain satisfies the detailed balance with respect to the probability $\pi_{N}$, i.e.,

$$
\pi_{N}\left(c_{a} g\right)=\pi_{N}\left(c_{a} g^{a}\right) .
$$

The operator $\mathcal{G}_{N}$ is selfadjoint in $L^{2}\left(S_{N}, d \pi_{N}\right)$, the corresponding Dirichlet form is

$$
\mathcal{E}_{N}(f, f):=\pi_{N}\left(f\left(-\mathcal{G}_{N}\right) f\right)=\frac{1}{2} \sum_{a \in B_{N}} \pi_{N}\left[c_{a}\left(\nabla_{a} f\right)^{2}\right] .
$$

We next show that for $\beta$ small enough the spectral gap of $\mathcal{G}_{N}^{\beta}$ is strictly positive uniformly in $N$. 
Theorem A.1. Assume $\sup _{N} \sup _{a}\left\|\nabla_{a} E_{N}\right\|_{\infty}<+\infty$. Then there exist $\beta_{0}, K_{0}>0$ such that for any $\beta \in\left[0, \beta_{0}\right]$ and any $N$

$$
\frac{1}{K_{0}} \leq \operatorname{gap}\left(\mathcal{G}_{N}^{\beta}\right) \leq K_{0} .
$$

Proof of the upper bound. In view of the variational characterization (2.7) of the spectral gap $\mathcal{G}_{N}$, it is enough to exhibit a suitable test function. We next show that by choosing $f(\sigma)=\mathbf{1}_{\{1\}}(\sigma(1))$, the upper bound in (A.4) follows.

The variance of $f$ is $\pi_{N}(f, f)=\pi_{N}(\sigma(1)=1)\left[1-\pi_{N}(\sigma(1)=1)\right]$. To compute the Dirichlet form, we first observe that $\nabla_{a} f_{N}$ vanishes if $1 \notin a$. On the other hand, if $a=\{1, y\}$ for some $y \in\{2, \ldots, N\}$

$$
\left[\nabla_{\{1, y\}} f_{N}(\sigma)\right]^{2}=\mathbf{1}_{\{1\}}(\sigma(1))+\mathbf{1}_{\{1\}}(\sigma(y)) .
$$

Whence, in view of (A.2),

$$
\begin{aligned}
\mathcal{E}_{N}(f, f) & =\frac{1}{2} \sum_{y} \pi_{N}\left(c_{\{1, y\}}\left[\mathbf{1}_{\{1\}}(\sigma(1))+\mathbf{1}_{\{1\}}(\sigma(y))\right]\right) \\
& \leq \exp \left\{\frac{\beta}{2} \sup _{N, a}\left\|\nabla_{a} E_{N}\right\|_{\infty}\right\} \pi_{N}(\sigma(1)=1) .
\end{aligned}
$$

Therefore

$$
\operatorname{gap}\left(\mathcal{G}_{N}\right) \leq \frac{\exp \left\{\frac{\beta}{2} \sup _{N, a}\left\|\nabla_{a} E_{N}\right\|_{\infty}\right\}}{1-\pi_{N}(\sigma(1)=1)} .
$$

It remains to show that the denominator above is bounded away from 0 uniformly in $N$. We claim that

$$
\frac{1}{N} \exp \left\{-\beta \sup _{N, a}\left\|\nabla_{a} E_{N}\right\|_{\infty}\right\} \leq \pi_{N}(\sigma(1)=1) \leq \frac{1}{N} \exp \left\{\beta \sup _{N, a}\left\|\nabla_{a} E_{N}\right\|_{\infty}\right\} .
$$

Indeed, fix $x \in V_{N}$ and observe

$$
\begin{aligned}
\pi_{N}(\sigma(1) & =1)=\frac{1}{Z_{N}} \sum_{\sigma} \pi_{N}(\sigma) \mathbf{1}_{\{1\}}(\sigma(1))=\frac{1}{Z_{N}} \sum_{\sigma} \pi_{N}\left(\sigma^{\{1, x\}}\right) \mathbf{1}_{\{1\}}(\sigma(x)) \\
& =\frac{1}{Z_{N}} \sum_{\sigma} \pi_{N}(\sigma) \frac{\pi_{N}\left(\sigma^{\{1, x\}}\right)}{\pi_{N}(\sigma)} \mathbf{1}_{\{1\}}(\sigma(x))=\pi_{N}\left(e^{-\beta \nabla_{\{1, x\}} E_{N}} \mathbf{1}_{\{1\}}(\sigma(x))\right) .
\end{aligned}
$$

This yields

$$
\exp \left\{-\beta \sup _{N, a}\left\|\nabla_{a} E_{N}\right\|_{\infty}\right\} \leq \frac{\pi_{N}(\sigma(x)=1)}{\pi_{N}(\sigma(1)=1)} \leq \exp \left\{\beta \sup _{N, a}\left\|\nabla_{a} E_{N}\right\|_{\infty}\right\} .
$$

Summing over $x \in V_{N}$ and observing that $\sum_{x} \pi_{N}(\sigma(x)=1)=1$ we get (A.5).

Proof of the lower bound. The proof is based on the $\Gamma_{2}$ approach, see e.g. 2], as adapted to the context of interacting particles systems in [5]. The starting point is the observation, which follows from the spectral theorem, that $\operatorname{gap}\left(\mathcal{G}_{N}\right)$ is the largest constant $k$ such that for any $f: S_{N} \rightarrow \mathbf{R}$

$$
\pi_{N}\left[\left(\mathcal{G}_{N} f\right)^{2}\right] \geq k \mathcal{E}_{N}(f, f)=\frac{k}{2} \sum_{a \in B_{N}} \pi_{N}\left[c_{a}\left(\nabla_{a} f\right)^{2}\right] .
$$


To prove the lower bound in (A.4) it is therefore enough to show there exist a constant $k$ independent of $N$ such that (A.6) holds. We proceed in two steps. We first show that

$$
\begin{aligned}
& \pi_{N}\left[\left(\mathcal{G}_{N} f\right)^{2}\right] \\
& \quad \geq \sum_{\substack{a, b \in B_{N} \\
a \cap b \neq \emptyset}} \pi_{N}\left[c_{a} c_{b} \nabla_{a} f \nabla_{b} f\right]+\frac{1}{2} \sum_{\substack{a, b \in B_{N} \\
a \cap b=\emptyset}} \pi_{N}\left[c_{a} c_{b}\left(1-\frac{c_{b}^{a}}{c_{b}}\right) \nabla_{a} f \nabla_{b} f\right] .
\end{aligned}
$$

Then we prove there exists a constant $k$ independent of $N$ such that

$$
\begin{aligned}
& \frac{k}{2} \sum_{a \in B_{N}} \pi_{N}\left[c_{a}\left(\nabla_{a} f\right)^{2}\right] \\
& \quad \leq \sum_{\substack{a, b \in B_{N} \\
a \cap b \neq \emptyset}} \pi_{N}\left[c_{a} c_{b} \nabla_{a} f \nabla_{b} f\right]+\frac{1}{2} \sum_{\substack{a, b \in B_{N} \\
a \cap b=\emptyset}} \pi_{N}\left[c_{a} c_{b}\left(1-\frac{c_{b}^{a}}{c_{b}}\right) \nabla_{a} f \nabla_{b} f\right] .
\end{aligned}
$$

While the inequality (A.7) can be obtained as a consequence of Corollary 2.3 and Proposition 2.4 in [5], we next give a direct proof in the present setting. Observe that

$$
\begin{aligned}
\pi_{N}\left[\left(\mathcal{G}_{N} f\right)^{2}\right] & =\sum_{a, b \in B_{N}} \pi_{N}\left[c_{a} c_{b} \nabla_{a} f \nabla_{b} f\right] \\
& =\sum_{a \cap b \neq \emptyset} \pi_{N}\left[c_{a} c_{b} \nabla_{a} f \nabla_{b} f\right]+\sum_{a \cap b=\emptyset} \pi_{N}\left[c_{a} c_{b} \nabla_{a} f \nabla_{b} f\right] .
\end{aligned}
$$

We rewrite the last term as

$$
\begin{aligned}
\sum_{a \cap b=\emptyset} & \pi_{N}\left[c_{a} c_{b} \nabla_{a} f \nabla_{b} f\right] \\
& =\frac{1}{2} \sum_{a \cap b=\emptyset} \pi_{N}\left[c_{a} c_{b}\left(1-\frac{c_{b}^{a}}{c_{b}}\right) \nabla_{a} f \nabla_{b} f\right]+\frac{1}{2} \sum_{a \cap b=\emptyset} \pi_{N}\left[c_{a} c_{b}\left(1+\frac{c_{b}^{a}}{c_{b}}\right) \nabla_{a} f \nabla_{b} f\right] .
\end{aligned}
$$

We claim that the last term on the right hand side above is positive. This statement together with (A.9) trivially implies (A.7). To prove the previous claim, fix $a, b \in$ $B_{N}$, with $a \cap b=\emptyset$ and observe that in this case $\left(\nabla_{a} f\right)^{a}\left(\nabla_{b} f\right)^{a}=-\nabla_{a} f \nabla_{b} f^{a}$. The detailed balance condition (A.3) now implies

$$
\begin{aligned}
& \pi_{N}\left[c_{a} c_{b}\left(1+\frac{c_{b}^{a}}{c_{b}}\right) \nabla_{a} f \nabla_{b} f\right]=\pi_{N}\left[c_{a}\left(c_{b}+c_{b}^{a}\right) \nabla_{a} f \nabla_{b} f\right] \\
& \quad=\pi_{N}\left[c_{a}\left(c_{b}^{a}+c_{b}\right)\left(\nabla_{a} f\right)^{a}\left(\nabla_{b} f\right)^{a}\right]=-\pi_{N}\left[c_{a}\left(c_{b}^{a}+c_{b}\right) \nabla_{a} f \nabla_{b} f^{a}\right] .
\end{aligned}
$$

Then

$$
\begin{aligned}
\pi_{N}\left[c_{a} c_{b}\left(1+\frac{c_{b}^{a}}{c_{b}}\right) \nabla_{a} f \nabla_{b} f\right] & =\frac{1}{2} \pi_{N}\left[c_{a}\left(c_{b}+c_{b}^{a}\right)\left(\nabla_{a} f \nabla_{b} f-\nabla_{a} f \nabla_{b} f^{a}\right)\right] \\
& =-\frac{1}{2} \pi_{N}\left[c_{a}\left(c_{b}+c_{b}^{a}\right) \nabla_{a} f \nabla_{a} \nabla_{b} f\right],
\end{aligned}
$$

Furthermore, by direct computation,

$$
c_{a}\left(c_{b}+c_{b}^{a}\right)=c_{b}\left(c_{a}+c_{a}^{b}\right) .
$$


By using (A.10), A.11), detailed balance (A.3), and (A.11) again we obtain

$$
\begin{aligned}
\pi_{N}[ & \left.c_{a} c_{b}\left(1+\frac{c_{b}^{a}}{c_{b}}\right) \nabla_{a} f \nabla_{b} f\right] \\
& =-\frac{1}{2} \pi_{N}\left[c_{a}\left(c_{b}+c_{b}^{a}\right) \nabla_{a} f \nabla_{a} \nabla_{b} f\right]=-\frac{1}{2} \pi_{N}\left[c_{b}\left(c_{a}+c_{a}^{b}\right) \nabla_{a} f \nabla_{a} \nabla_{b} f\right] \\
& =\frac{1}{2} \pi_{N}\left[c_{b}\left(c_{a}+c_{a}^{b}\right) \nabla_{a} f^{b} \nabla_{a} \nabla_{b} f\right]=\frac{1}{2} \pi_{N}\left[c_{a}\left(c_{b}+c_{b}^{a}\right) \nabla_{a} f^{b} \nabla_{a} \nabla_{b} f\right] .
\end{aligned}
$$

By averaging the previous equation and A.10 we get

$$
\begin{aligned}
\pi_{N}\left[c_{a} c_{b}\left(1+\frac{c_{b}^{a}}{c_{b}}\right) \nabla_{a} f \nabla_{b} f\right] & =\frac{1}{4} \pi_{N}\left[c_{a}\left(c_{b}+c_{b}^{a}\right)\left(\nabla_{a} f^{b}-\nabla_{a} f\right) \nabla_{a} \nabla_{b} f\right] \\
& =\frac{1}{4} \pi_{N}\left[c_{a}\left(c_{b}+c_{b}^{a}\right)\left(\nabla_{a} \nabla_{b} f\right)^{2}\right] \geq 0
\end{aligned}
$$

which concludes the proof of the claim.

In order to prove (A.8) we observe that

$$
\sum_{a \cap b \neq \emptyset} \pi_{N}\left[c_{a} c_{b} \nabla_{a} f \nabla_{b} f\right]=\sum_{a} \pi_{N}\left[c_{a}^{2}\left(\nabla_{a} f\right)^{2}\right]+\sum_{\substack{a \cap b \neq \emptyset \\ a \neq b}} \pi_{N}\left[c_{a} c_{b} \nabla_{a} f \nabla_{b} f\right] .
$$

Furthermore, given $a, b \in B_{N}$ such that $a \cap b \neq \emptyset$ and $a \neq b$ there exists a unique triangle $T$ such that $a, b \in T$. A triangle here is an element of

$$
\mathcal{T}_{N}:=\left\{\{a, b, c\} \subset B_{N}:|\{a, b, c\}|=3, a \cap b \neq \emptyset, a \cap c \neq \emptyset, b \cap c \neq \emptyset\right\} .
$$

Therefore

$$
\sum_{\substack{a \cap b \neq \emptyset \\ a \neq b}} \pi_{N}\left[c_{a} c_{b} \nabla_{a} f \nabla_{b} f\right]=\sum_{T \in \mathcal{T}_{N}} \sum_{\substack{a, b \in T \\ a \neq b}} \pi_{N}\left[c_{a} c_{b} \nabla_{a} f \nabla_{b} f\right]
$$

Note that

$$
\begin{aligned}
\sum_{T \in \mathcal{T}_{N}} & \sum_{\substack{a, b \in T \\
a \neq b}} \pi_{N}\left[c_{a} c_{b} \nabla_{a} f \nabla_{b} f\right] \\
& =\sum_{T \in \mathcal{T}_{N}} \sum_{a, b \in T} \pi_{N}\left[c_{a} c_{b} \nabla_{a} f \nabla_{b} f\right]-\sum_{T \in \mathcal{T}_{N}} \sum_{a \in T} \pi_{N}\left[c_{a}^{2}\left(\nabla_{a} f\right)^{2}\right] \\
& =\sum_{T \in \mathcal{T}_{N}} \sum_{a, b \in T} \pi_{N}\left[c_{a} c_{b} \nabla_{a} f \nabla_{b} f\right]-\sum_{a}\left|\left\{T \in \mathcal{T}_{N}: T \ni a\right\}\right| \pi_{N}\left[c_{a}^{2}\left(\nabla_{a} f\right)^{2}\right] \\
& =\sum_{T \in \mathcal{T}_{N}} \sum_{a, b \in T} \pi_{N}\left[c_{a} c_{b} \nabla_{a} f \nabla_{b} f\right]-(N-2) \sum_{a} \pi_{N}\left[c_{a}^{2}\left(\nabla_{a} f\right)^{2}\right] .
\end{aligned}
$$

By plugging this result in $(\mathrm{A} .12$ we get

$$
\sum_{a \cap b \neq \emptyset} \pi_{N}\left[c_{a} c_{b} \nabla_{a} f \nabla_{b} f\right]=\sum_{T \in \mathcal{T}_{N}} \sum_{a, b \in T} \pi_{N}\left[c_{a} c_{b} \nabla_{a} f \nabla_{b} f\right]-(N-3) \sum_{a} \pi_{N}\left[c_{a}^{2}\left(\nabla_{a} f\right)^{2}\right] .
$$

For any $T \in \mathcal{T}_{N}$ define the set of vertexes of $T$ as $\widetilde{T}:=\bigcup_{a \in T} a$. Then

$$
\sum_{a, b \in T} \pi_{N}\left[c_{a} c_{b} \nabla_{a} f \nabla_{b} f\right]=\pi_{N}\left[\sum_{a, b \in T} \pi_{N}\left[c_{a} c_{b} \nabla_{a} f \nabla_{b} f \mid \sigma(z): z \notin \widetilde{T}\right]\right] .
$$


We prove in Lemma A.2 below that there exists a constant $C_{1}(\beta)>0$ satisfying $\lim _{\beta \downarrow 0} C_{1}(\beta)=1$ such that for any $N \geq 3$, any $f: S_{N} \rightarrow \mathbf{R}$, and any $\sigma \in S_{N}$

$$
\frac{N}{3} \sum_{a, b \in T} \pi_{N}\left[c_{a} c_{b} \nabla_{a} f \nabla_{b} f \mid \sigma(z): z \notin \widetilde{T}\right] \geq \frac{C_{1}(\beta)}{2} \sum_{a \in T} \pi_{N}\left[c_{a}\left(\nabla_{a} f\right)^{2} \mid \sigma(z): z \notin \widetilde{T}\right] .
$$

By plugging this bound into (A.13), we deduce

$$
\begin{aligned}
\sum_{a \cap b \neq \emptyset} & \pi_{N}\left[c_{a} c_{b} \nabla_{a} f \nabla_{b} f\right] \\
& \geq \frac{3 C_{1}(\beta)}{2 N} \sum_{T \in \mathcal{T}_{N}} \sum_{a \in T} \pi_{N}\left[c_{a}\left(\nabla_{a} f\right)^{2}\right]-(N-3) \sum_{a} \pi_{N}\left[c_{a}^{2}\left(\nabla_{a} f\right)^{2}\right] \\
& =\frac{3 C_{1}(\beta)(N-2)}{2 N} \sum_{a} \pi_{N}\left[c_{a}\left(\nabla_{a} f\right)^{2}\right]-(N-3) \sum_{a} \pi_{N}\left[c_{a}^{2}\left(\nabla_{a} f\right)^{2}\right] \\
& \geq\left(\frac{3 C_{1}(\beta)(N-2)}{2 N}-(N-3) \sup _{a}\left\|c_{a}\right\|_{\infty}\right) \sum_{a} \pi_{N}\left[c_{a}\left(\nabla_{a} f\right)^{2}\right] .
\end{aligned}
$$

In view of (A.2),

$$
\sup _{a}\left\|c_{a}\right\|_{\infty} \leq \frac{1}{N} \exp \left\{\frac{\beta}{2} \sup _{N, a}\left\|\nabla_{a} E_{N}\right\|_{\infty}\right\} .
$$

Recalling the hypotheses $\sup _{N} \sup _{a}\left\|\nabla_{a} E_{N}\right\|_{\infty}<+\infty$, from (A.14) we then deduce there exists a constant $C_{2}(\beta)>0$ satisfying $\lim _{\beta \downarrow 0} C_{2}(\beta)=1$ such that

$$
\sum_{a \cap b \neq \emptyset} \pi_{N}\left[c_{a} c_{b} \nabla_{a} f \nabla_{b} f\right] \geq \frac{C_{2}(\beta)}{2} \sum_{a} \pi_{N}\left[c_{a}\left(\nabla_{a} f\right)^{2}\right] .
$$

To conclude the proof of (A.8) we show that the second term on its right hand side is, for $\beta$ small enough, of order $\beta$. By Schwarz inequality and A.11

$$
\begin{aligned}
& \left|\frac{1}{2} \sum_{\substack{a, b \in B_{N} \\
a \cap b=\emptyset}} \pi_{N}\left[c_{a} c_{b}\left(1-\frac{c_{b}^{a}}{c_{b}}\right) \nabla_{a} f \nabla_{b} f\right]\right| \leq \frac{1}{2} \sum_{a \cap b=\emptyset} \pi_{N}\left[c_{a} c_{b}\left|1-\frac{c_{b}^{a}}{c_{b}}\right|\left|\nabla_{a} f\right|\left|\nabla_{b} f\right|\right] \\
& \quad \leq \frac{1}{4}\left\{\sum_{a \cap b=\emptyset} \pi_{N}\left[c_{a} c_{b}\left|1-\frac{c_{b}^{a}}{c_{b}}\right|\left(\nabla_{a} f\right)^{2}\right]+\sum_{a \cap b=\emptyset} \pi_{N}\left[c_{a} c_{b}\left|1-\frac{c_{b}^{a}}{c_{b}}\right|\left(\nabla_{b} f\right)^{2}\right]\right\} \\
& \quad=\frac{1}{2} \sum_{a \cap b=\emptyset} \pi_{N}\left[c_{a} c_{b}\left|1-\frac{c_{b}^{a}}{c_{b}}\right|\left(\nabla_{a} f\right)^{2}\right]=\frac{1}{2} \sum_{a} \pi_{N}\left[c_{a}\left(\nabla_{a} f\right)^{2} \sum_{b: b \cap a=\emptyset} c_{b}\left|1-\frac{c_{b}^{a}}{c_{b}}\right|\right] .
\end{aligned}
$$

The hypotheses of the theorem implies $\sup _{N} \sup _{a, b}\left\|\nabla_{a} \nabla_{b} E_{N}\right\|_{\infty}<+\infty$. Recalling (A.15), for $\beta$ small enough we then have

$$
\begin{aligned}
\sum_{b: b \cap a=\emptyset} c_{b}\left|1-\frac{c_{b}^{a}}{c_{b}}\right| & \leq \sup _{a}\left\|c_{a}\right\|_{\infty} \sum_{b: b \cap a=\emptyset}\left|1-e^{-\frac{\beta}{2} \nabla_{a} \nabla_{b} E_{N}}\right| \\
& \leq \beta \sup _{a}\left\|c_{a}\right\|_{\infty} \sum_{b: b \cap a=\emptyset}\left|\nabla_{a} \nabla_{b} E_{N}\right| \leq C_{3} \beta
\end{aligned}
$$

for some constant $C_{3}$ independent of $N$. Therefore

$$
\frac{1}{2} \sum_{\substack{a, b \in B_{N} \\ a \cap b=\emptyset}} \pi_{N}\left[c_{a} c_{b}\left(1-\frac{c_{b}^{a}}{c_{b}}\right) \nabla_{a} f \nabla_{b} f\right] \geq-\frac{C_{3} \beta}{2} \sum_{a} \pi_{N}\left[c_{a}\left(\nabla_{a} f\right)^{2}\right],
$$


which together with (A.16) completes the proof of A.8).

Lemma A.2. Assume $\sup _{N} \sup _{a}\left\|\nabla_{a} E_{N}\right\|_{\infty}<+\infty$. Then there exists a constant $C_{1}(\beta)$ satisfying $\lim _{\beta \downarrow 0} C_{1}(\beta)=1$ such that for any $N \geq 3$, any $T \in \mathcal{T}_{N}$, any $f: S_{N} \rightarrow \mathbf{R}$, and any $\sigma \in S_{N}$

$$
\frac{N}{3} \sum_{a, b \in T} \pi_{N}\left[c_{a} c_{b} \nabla_{a} f \nabla_{b} f \mid \sigma(z): z \notin \widetilde{T}\right] \geq \frac{C_{1}(\beta)}{2} \sum_{a \in T} \pi_{N}\left[c_{a}\left(\nabla_{a} f\right)^{2} \mid \sigma(z): z \notin \widetilde{T}\right]
$$

where we recall $\widetilde{T}:=\bigcup_{a \in T} a$.

Proof. The argument relies on two ingredients. The first is that, given a triangle $T \in \mathcal{T}_{N}$, the conditional probability $\pi_{N}^{\beta}[\cdot \mid \sigma(z): \notin \widetilde{T}]$ is, for $\beta$ small enough, close to the uniform measure. Namely, there exist $C_{4}(\beta)$ satisfying $\lim _{\beta \downarrow 0} C_{4}(\beta)=1$ independent of $N, T \in \mathcal{T}_{N}$, and $\sigma \in S_{N}$, such that

$$
\frac{1}{C_{4}(\beta)} \leq \frac{\pi_{N}^{\beta}[\sigma \mid \sigma(z): z \notin \widetilde{T}]}{\pi_{N}^{0}[\sigma \mid \sigma(z): z \notin \widetilde{T}]} \leq C_{4}(\beta) .
$$

The second ingredient is that the spectral gap of the interchange process on a graph with 3 vertices is equal to 1 . This statement readily implies

$$
\left.\pi_{N}^{0}[f, f \mid \sigma(z): z \notin \widetilde{T}]\right] \leq \frac{1}{6} \sum_{a \in T} \pi_{N}^{0}\left[\left(\nabla_{a} f\right)^{2} \mid \sigma(z): z \notin \widetilde{T}\right] .
$$

We first show that A.17) and A.18) imply the thesis. Since $\pi_{N}^{0}(\cdot \mid \sigma(z): z \notin \widetilde{T})$ is the uniform measure on a set of cardinality 6 , in view of (A.18)

$$
\begin{aligned}
\pi_{N}^{\beta}[f, f \mid \sigma(z): z \notin \widetilde{T}] \leq C_{4}(\beta)^{2} \pi_{N}^{0}[f, f \mid \sigma(z): z \notin \widetilde{T}] \\
\quad \leq \frac{C_{4}(\beta)^{2}}{6} \sum_{a \in T} \pi_{N}^{0}\left[\left(\nabla_{a} f\right)^{2} \mid \sigma(z): z \notin \widetilde{T}\right] \\
\quad \leq \frac{1}{3} C_{4}(\beta)^{3} N e^{\frac{\beta}{2} \sup _{N, a}\left\|\nabla_{a} E_{N}\right\|_{\infty}} \frac{1}{2} \sum_{a \in T} \pi_{N}^{\beta}\left[c_{a}\left(\nabla_{a} f\right)^{2} \mid \sigma(z): z \notin \widetilde{T}\right] .
\end{aligned}
$$

Whence, by using the characterization of the spectral gap given in (A.6),

$$
\begin{aligned}
& \sum_{a, b \in T} \pi_{N}^{\beta}\left[c_{a} c_{b} \nabla_{a} f \nabla_{b} f \mid \sigma(z): z \notin \widetilde{T}\right] \\
& \quad \geq \frac{3}{C_{4}(\beta)^{3} N} \exp \left\{-\frac{\beta}{2} \sup _{N, a}\left\|\nabla_{a} E_{N}\right\|_{\infty}\right\} \frac{1}{2} \sum_{a \in T} \pi_{N}^{\beta}\left[c_{a}\left(\nabla_{a} f\right)^{2} \mid \sigma(z): z \notin \widetilde{T}\right]
\end{aligned}
$$

which, for a suitable $C_{1}(\beta)$, is the thesis of the lemma.

The estimate (A.17) follows from standard arguments. Firstly note that for any $a \in T$

$$
\frac{\pi_{N}^{\beta}\left[\sigma^{a} \mid \sigma(z): z \notin \widetilde{T}\right]}{\pi_{N}^{\beta}[\sigma \mid \sigma(z): z \notin \widetilde{T}]}=\exp \left\{-\beta \nabla_{a} E_{N}(\sigma)\right\} .
$$

Therefore, by observing that any two given permutations in $S_{3}$ can be connected at most by two transpositions, a telescopic argument yields that for any $\sigma, \sigma^{\prime} \in S_{N}$ such that $\sigma(z)=\sigma^{\prime}(z)$ for $z \notin \widetilde{T}$

$$
\exp \left\{-2 \beta \sup _{N, a}\left\|\nabla_{a} E_{N}\right\|_{\infty}\right\} \leq \frac{\pi_{N}^{\beta}\left[\sigma^{\prime} \mid \sigma^{\prime}(z): z \notin \widetilde{T}\right]}{\pi_{N}^{\beta}[\sigma \mid \sigma(z): z \notin \widetilde{T}]} \leq \exp \left\{2 \beta \sup _{N, a}\left\|\nabla_{a} E_{N}\right\|_{\infty}\right\} .
$$


By averaging the above inequality over $\sigma^{\prime}$ the bound A.17) follows.

The spectral gap of the interchange process on $\{1,2,3\}$ can be deduced from the general results in [8, 11]. An elementary proof can however also be obtained by writing out the $6 \times 6$ matrix corresponding to the generator and computing its eigenvalues, as in the example at page 50 of 12 . We order the 6 permutations of $S_{3}$ as $\left(\begin{array}{l}123 \\ 123\end{array}\right),\left(\begin{array}{l}123 \\ 132\end{array}\right),\left(\begin{array}{l}123 \\ 231\end{array}\right),\left(\begin{array}{l}123 \\ 213\end{array}\right),\left(\begin{array}{l}123 \\ 312\end{array}\right)$, and $\left(\begin{array}{l}123 \\ 321\end{array}\right)$. With this choice, the generator of the interchange process is represented by the matrix

$$
\left(\begin{array}{rrrrrr}
-1 & \frac{1}{3} & 0 & \frac{1}{3} & 0 & \frac{1}{3} \\
\frac{1}{3} & -1 & \frac{1}{3} & 0 & \frac{1}{3} & 0 \\
0 & \frac{1}{3} & -1 & \frac{1}{3} & 0 & \frac{1}{3} \\
\frac{1}{3} & 0 & \frac{1}{3} & -1 & \frac{1}{3} & 0 \\
0 & \frac{1}{3} & 0 & \frac{1}{3} & -1 & \frac{1}{3} \\
\frac{1}{3} & 0 & \frac{1}{3} & 0 & \frac{1}{3} & -1
\end{array}\right)
$$

whose eigenvalues are 0 (simple), -1 (with multiplicity four), and -2 (simple).

We finally show, as a corollary of the previous result, that the spectral gap of the $\mathrm{ABC}$ dynamics on the complete graph is of order one.

Proof of Lemma 4.1. Fix $N$ multiple of three and let $\chi_{N}: S_{N} \rightarrow \Omega_{N}$ be the projection defined by

$$
\left(\chi_{N} \sigma\right)(x):=\left\{\begin{array}{lll}
A & \text { if } \sigma(x) \equiv 1 & \bmod 3 \\
B & \text { if } \sigma(x) \equiv 2 & \bmod 3 \\
C & \text { if } \sigma(x) \equiv 3 & \bmod 3 .
\end{array}\right.
$$

Namely, $\chi_{N}$ resolves only three out of the original $N$ colors. Recalling (2.5), let $E_{N}: S_{N} \rightarrow \mathbf{R}$ be defined by $E_{N}:=N H_{N} \circ \chi_{N}$. For this choice the ABC dynamics with long jumps, i.e. the process with the generator (4.2), can be realized as the $\chi_{N}$-projection of the process with generator (A.1). In particular, $\nu_{N}^{\beta}=\pi_{N}^{\beta} \circ \chi_{N}^{-1}$ and $\operatorname{gap}\left(\mathcal{L}_{N}^{\beta}\right) \geq \operatorname{gap}\left(\mathcal{G}_{N}^{\beta}\right)$. Since $H_{N} \circ \chi_{N}$ satisfies the hypotheses in Theorem A.1. the statement follows.

Acknowledgements. We express our warmest thanks to C. Furtlehner who pointed out to us the possible occurrence of a metastable behavior for the ABC model. We also thank T. Bodineau and J.L. Lebowitz for useful discussions. N.C. and G.P. acknowledges the financial support of the European Research Council through the "Advanced Grant" PTRELSS 228032.

\section{REFERENCES}

[1] Ayyer A., Carlen E.A., Lebowitz J.L., Mohanty P.K., Mukamel D., Speer E.R.; Phase diagram of the ABC model on an interval. J. Stat. Phys. 137, 1166-1204 (2009).

[2] Bakry D., Emery M.; Diffusions hypercontractives, in Séminaire de Probabilités XIX, Lecture Notes in Mathematics 1123, 177-206. Springer, Berlin, 1985.

[3] Bodineau T., Derrida B.; Phase fluctuations in the ABC model. Preprint 2011.

[4] Bodineau T., Derrida B., Lecomte V., van Wijland F.; Long range correlations and phase transitions in Non-equilibrium diffusive systems. J. Stat. Phys. 133, 1013-1031 (2008).

[5] Boudou A.-S., Caputo P., Dai Pra P., Posta G.; Spectral gap estimates for interacting particle systems via a Bochner-type identity. J. Funct. Anal. 232, 222-258 (2006).

[6] Cancrini N., Cesi F., Martinelli F.; The spectral gap for the Kawasaki dynamics at low temperature. J. Statist. Phys. 95, 215-271 (1999).

[7] Cancrini N., Martinelli F.; On the spectral gap of Kawasaki dynamics under a mixing condition revisited. J. Math. Phys. 41, 1391-1423 (2000). 
[8] Caputo P., Liggett T.M., Richthammer T.; Proof of Aldous' spectral gap conjecture. J. Amer. Math. Soc. 23, 831-851 (2010).

[9] Clincy M., Derrida B., Evans M.R.; Phase transition in the ABC model. Phys. Rev. E 67, $066115(2003)$

[10] Dembo A., Zeitouni O.; Large Deviations Techniques and Applications. Second edition. Springer, New York, 1998

[11] Diaconis P., Shahshahani M.; Generating a random permutation with random transpositions. Z. Wahrsch. Verw. Gebiete 57, 159-179 (1981).

[12] Diaconis P.; Group representations in probability and statistics. Institute of Mathematical Statistics Lecture Notes - Monograph Series, 11. Institute of Mathematical Statistics, Hayward, 1988

[13] Ellis R.S.; Entropy, large deviations, and statistical mechanics. Springer, New York, 1985.

[14] Evans M.R., Kafri Y., Koduvely H.M., Mukamel D.; Phase separation in one-dimensional driven diffusive systems. Phys. Rev. Lett. 80, 425-429 (1998).

[15] Evans M.R., Kafri Y., Koduvely H.M., Mukamel D.; Phase separation and coarsening in onedimensional driven diffusive systems: Local dynamics leading to long-range Hamiltonians. Phys. Rev. E 58, 2764-2778 (1998).

[16] Fayolle G., Furtlehner C.; Stochastic deformations of sample paths of random walks and exclusion models. Mathematics and computer science. III, 415-428, Trends Math., Birkhäuser, Basel, 2004.

[17] Fayolle G., Furtlehner C.; Stochastic dynamics of discrete curves and multi-type exclusion processes. J. Stat. Phys. 127, 1049-1094 (2007).

[18] Furtlehner C.; Private communication 2011.

[19] Jensen L.H.; Large deviations of the asymmetric simple exclusion process in one dimension. Ph.D. Thesis, Courant Institute NYU, 2000.

[20] Kipnis C., Landim C.; Scaling limits of interacting particle systems. Springer, Berlin, 1999.

[21] Lanford O.E.; Entropy and Equilibrium States is Classical Statistical Mechanics. Lenard A., ed., Lecture Notes in Physics, 20. Springer, Berlin, 1973.

[22] Lu S.L., Yau H-T; Spectral gap and logarithmic Sobolev inequality for Kawasaki and Glauber dynamics. Commun. Math. Phys. 156, 399-433 (1993).

[23] Petrov V.V.; Sums of independent random variables. Springer-Verlag, New York-Heidelberg, 1975.

[24] Quastel J.; Diffusion of color in the simple exclusion process. Comm. Pure Appl. Math. 45, 623-679 (1992)

LORENZO BERTINI

Dipartimento di Matematica, Università di Roma 'LA SAPienza',

P.le Aldo Moro 5, 00185 Roma, Italy

E-mail address: bertini@mat.uniroma1.it

Nicoletta CANCRini

Dipartimento di Matematica Pura e Applicata, Università dell'Aquila, 67100 Coppito, L'Aquila, Italy

E-mail address: nicoletta.cancrini@univaq.it

Gustavo Posta

Dipartimento di Matematica, Politecnico di Milano

P.za Leonardo da Vinci 32, 20133 Milano, Italy

E-mail address: gustavo.posta@polimi.it 